\title{
Skew characters and cyclic sieving
}

\author{
Per Alexandersson ${ }^{1}$, Stephan Pfannerer ${ }^{2}$, Martin Rubey ${ }^{2}$ and Joakim Uhlin $^{3}$ \\ ${ }^{1}$ Department of Mathematics, Stockholm University, S-10691, Stockholm, Sweden; E-mail: per.w.alexandersson@gmail.com. \\ ${ }^{2}$ Fakultät für Mathematik und Geoinformation, TU Wien, Austria; E-mail: stephan.pfannerer@tuwien.ac.at, \\ martin.rubey@tuwien.ac.at. \\ ${ }^{3}$ Department of Mathematics, Stockholm University, S-10691, Stockholm, Sweden; E-mail: joakim_uhlin@hotmail.com.
}

Received: 12 May 2020; Revised: 30 December 2020; Accepted: 22 January 2021

2020 Mathematics Subject Classification: Primary - 05E10; Secondary - 05E05

\begin{abstract}
In 2010, Rhoades proved that promotion on rectangular standard Young tableaux, together with the associated fake-degree polynomial, provides an instance of the cyclic sieving phenomenon. We extend this result to $m$-tuples of skew standard Young tableaux of the same shape, for fixed $m$, subject to the condition that the $m$ th power of the associated fake-degree polynomial evaluates to nonnegative integers at roots of unity. However, we are unable to specify an explicit group action. Put differently, we determine in which cases the $m$ th tensor power of a skew character of the symmetric group carries a permutation representation of the cyclic group.

To do so, we use a method proposed by Amini and the first author, which amounts to establishing a bound on the number of border-strip tableaux of skew shape. Finally, we apply our results to the invariant theory of tensor powers of the adjoint representation of the general linear group. In particular, we prove the existence of a bijection between permutations and Stembridge's alternating tableaux, which intertwines rotation and promotion.
\end{abstract}

\section{Introduction}

We determine which tensor powers of a skew character $\chi^{\lambda / \mu}$ of the symmetric group $\mathfrak{S}_{n}$ carry a permutation representation of the cyclic group of order $n$.

This problem can be rephrased in terms of Reiner, Stanton and White's cyclic sieving phenomenon [RSW04]. Let $\operatorname{SYT}(\lambda / \mu)$ be the set of standard Young tableaux of skew shape $\lambda / \mu$, and let $f^{\lambda / \mu}(q)$ be Lusztig's fake-degree polynomial for $\chi^{\lambda / \mu}$. Then there exists an action $\rho$ of the cyclic group of order $n=|\lambda / \mu|$ such that

$$
(\underbrace{\operatorname{SYT}(\lambda / \mu) \times \cdots \times \operatorname{SYT}(\lambda / \mu)}_{m},\langle\rho\rangle, f^{\lambda / \mu}(q)^{m})
$$

exhibits the cyclic sieving phenomenon if and only if $f^{\lambda / \mu}(q)^{m}$ evaluates to nonnegative integers at $n$th roots of unity. If $m$ is even, this is always the case. If $m$ is odd, it is the case if and only if every tiling of $\lambda / \mu$ with border strips of size $k$ has even height for every $k \mid n$ (see Theorem 44).

(C) The Author(s), 2021. Published by Cambridge University Press. This is an Open Access article, distributed under the terms of the Creative Commons Attribution licence (http://creativecommons.org/licenses/by/4.0/), which permits unrestricted re-use, distribution, and reproduction in any medium, provided the original work is properly cited. 
We also show that for any skew shape $\lambda / \mu$ and any integer $s>0$, there is an action $\tau$ of the cyclic group of order $s$ on stretched shapes such that

$$
\left(\operatorname{SYT}(s \lambda / s \mu),\langle\tau\rangle, f^{s \lambda / s \mu}(q)\right)
$$

exhibits the cyclic sieving phenomenon (see Theorem 49).

At this point we are unable to present $\rho$ and $\tau$ explicitly for general skew shapes $\lambda / \mu$. Instead, we use a characterisation of Alexandersson and Amini [AA19] which says that $f \in \mathbb{N}_{0}[q]$ is a cyclic sieving polynomial for a group action of the cyclic group of order $n$ if and only if for a primitive $n$th root of unity $\xi$ and all $k \mid n$, we have that $f\left(\xi^{k}\right) \in \mathbb{N}_{0}$ and

$$
\sum_{d \mid k} \mu(k / d) f\left(\xi^{d}\right) \geq 0,
$$

where $\mu$ is the number-theoretic Möbius function.

To apply this result, we establish a new bound for the absolute value of the skew character evaluated at a power of the long cycle. More precisely, Theorem 31 implies that for any $k \mid n$,

$$
\left|f^{\lambda / \mu}\left(\xi^{k}\right)\right| \geq \sum_{d \mid k, d<k}\left|f^{\lambda / \mu}\left(\xi^{d}\right)\right|,
$$

provided $\left|f^{\lambda / \mu}\left(\xi^{k}\right)\right| \geq 2$.

To prove this inequality, we show that

$$
\left|f^{\lambda / \mu}\left(\xi^{d}\right)\right|=\left|\chi^{\lambda / \mu}\left(\left(m^{d}\right)\right)\right|=|\operatorname{BST}(\lambda / \mu, m)|,
$$

which is the number of border-strip tableaux of shape $\lambda / \mu$ with strips of size $m$, extending the theorems for straight shapes of Springer [Spr74] and James and Kerber [JK84]. We then approximate the number of border-strip tableaux using a bound by Fomin and Lulov [FL97].

Our main motivation is an implication for the invariant theory of the general linear group, as we now explain. Let $\mathfrak{g l}_{r}$ be the adjoint representation of $\mathrm{GL}_{r}$, and consider its $n$th tensor power $\mathfrak{g l}_{r}^{\otimes n}$. The symmetric group $\mathfrak{S}_{n}$ acts on this space by permuting tensor positions. Thus, using Schur-Weyl duality, we can determine the subspace of $\mathrm{GL}_{n}$-invariants of $\mathfrak{g l}_{r}^{\otimes n}$, regarded as a representation of $\mathfrak{S}_{n}$. This representation turns out to be isomorphic to

$$
\bigoplus_{\substack{\lambda \vdash n \\ \ell(\lambda) \leq r}} S_{\lambda} \otimes S_{\lambda}
$$

where the direct sum is over all partitions of $n$ into at most $r$ parts, and $S_{\lambda}$ is the irreducible representation of $\mathfrak{S}_{n}$ corresponding to $\lambda$. In particular, for $r \geq n$, the dimension of the space of invariants equals the size of $\mathfrak{\Im}_{n}$.

A fundamental question of invariant theory is to find an explicit basis of the space of invariants which, if possible, enjoys further desirable properties. One such property is invariance under rotation of tensor positions, following Kuperberg's idea of web bases [Kup96].

An elegant and useful solution would be to describe a set of permutations in $\mathfrak{S}_{n}$ and a bijection from these to the basis elements which intertwines rotation of permutations (that is, conjugation with the long cycle) and rotation of tensor positions. It would be even nicer if this set of permutations for the invariants of $\mathfrak{g l}_{r}^{\otimes n}$ were a subset of the set of permutations for the invariants of $\mathfrak{g l} \mathfrak{l}_{r+1}^{\otimes n}$.

Although it appears to be difficult to exhibit such an intertwining bijection explicitly, our results, combined with previous work of Pfannerer, Rubey and Westbury [PRW20], imply that such a solution must exist (see Theorem 61). 
The existence of such an intertwining bijection is closely related to the existence of a rotation-invariant statistic st mapping permutations to partitions, such that $\left|\left\{\sigma \in \mathfrak{S}_{n}: \operatorname{st}(\sigma)=\lambda\right\}\right|=|\operatorname{SYT}(\lambda) \times \operatorname{SYT}(\lambda)|$ (see Corollary 56).

\subsection{Outline of the paper}

In Section 2 we recall the definition of the cyclic sieving phenomenon and establish the connection with characters of cyclic group actions. In Sections 3 to 6 we generalise Springer's theorem to skew shapes and show - using the Murnaghan-Nakayama rule, the abacus of James and Kerber and the Littlewood map - that the character evaluation of a skew character is, up to sign, equal to a certain number of border-strip tableaux. We stress that these identities are known for the straight-shape case. However, they are somewhat underappreciated gems which deserve more attention.

In Section 7 we provide the crucial bound on the number of border-strip tableaux of given shape, building on the approximation of Fomin and Lulov. In Section 8 we use this bound and the characterisation of Alexandersson and Amini to prove the existence of the group actions announced for skew standard tableaux.

Finally, in Section 9 we apply our results to permutations and the invariant theory of the adjoint representation of the general linear group.

\section{Cyclic group actions and cyclic sieving}

In this section we recall Reiner, Stanton and White's cyclic sieving phenomenon, characters of cyclic group actions and a result of Alexandersson and Amini characterising characters arising from cyclic group actions. We also recall Brauer's permutation lemma, which guarantees that two actions of the cyclic group which have the same character as linear representations are even isomorphic as group actions.

Definition 1 ([RSW04]). Let $X$ be a finite set and let $\rho$ be a generator of an action of the cyclic group of order $n$ on $X$.

Given a polynomial $f(q) \in \mathbb{N}_{0}[q]$, we say that the triple $(X,\langle\rho\rangle, f(q))$ exhibits the cyclic sieving phenomenon if for all $d \in \mathbb{Z}$,

$$
\#\left\{x \in X: \rho^{d} \cdot x=x\right\}=f\left(\xi^{d}\right),
$$

where $\xi$ is a primitive $n$th root of unity. In this case, $f(q)$ is a cyclic sieving polynomial for the group action.

In particular, the cardinality of $X$ is given by $f(1)$. More generally, realising the cyclic group of order $n$ as the group of $n$th roots of unity and identifying its ring of characters with $\mathbb{Z}[q] /\left(q^{n}-1\right)$, the cyclic sieving polynomial $f(q)$ modulo $q^{n}-1$ reduces to the character of the group action.

The cyclic sieving phenomenon owes its name to the fact that, mysteriously often, the most natural $q$-analogue of the counting formula for the cardinality of $X$ as a function of $n$ is a cyclic sieving polynomial. In many cases, the only known way to prove that a given $q$-analogue indeed is a cyclic sieving polynomial is to enumerate the number of fixed points of the group action and verify that the evaluation of the polynomial yields the same number.

Remark 2. If $(X,\langle\rho\rangle, f(q))$ exhibits the cyclic sieving phenomenon, then so does $\left(X,\left\langle\rho^{k}\right\rangle, f(q)\right)$ for any positive integer $k$ which restricts the group action to a subgroup. Moreover, for any positive integer $m$, the triple $\left(X^{m},\langle\rho\rangle, f(q)^{m}\right)$, where $\langle\rho\rangle$ acts on $X^{m}$ via $\rho \cdot\left(x_{1}, \ldots, x_{m}\right)=\left(\rho \cdot x_{1}, \ldots, \rho \cdot x_{m}\right)$, also exhibits the cyclic sieving phenomenon.

Much attention has been given to proving cyclic sieving phenomena on certain families of tableaux (see Table 1). Most famously, Rhoades has shown that SYT $\left(a^{b}\right)$ exhibits the cyclic sieving phenomenon, where the group action is promotion and the cyclic sieving polynomial is the fake-degree polynomial 
Table 1. Summary of known cyclic sieving phenomena on various sets of tableaux.

\begin{tabular}{lllc}
\hline Set & Group action & Statistic/ $f(q)$ & Reference \\
\hline $\operatorname{SYT}\left(a^{b}\right)$ & Promotion & maj & [Rho10a] \\
$\operatorname{SYT}\left(\left(n-m, 1^{m}\right)\right)$ & Promotion $^{\mathrm{a}}$ & $\left.\begin{array}{c}n-1 \\
m\end{array}\right]_{q}$ & [BMS14] \\
$\operatorname{SYT}(\lambda)$ & Evacuation $^{\mathrm{b}}$ & $\mathrm{maj}^{\mathrm{j}}$ & [Ste96] \\
$\operatorname{SSYT}\left(a^{b}, k\right)$ & $k$-promotion & $q^{-\kappa(\lambda)} \mathrm{s}_{a^{b}}\left(1, q, \ldots, q^{k-1}\right)$ & [Rho10a] \\
$\operatorname{COF}(n \lambda / n \mu)$ & Cyclic shift & a variant of maj & [AU20] \\
\hline
\end{tabular}

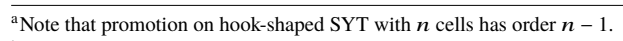

${ }^{\mathrm{b}}$ Evacuation is an involution, so the cyclic group has order 2.

$f^{\lambda}(q)$ associated with $\lambda=\left(a^{b}\right)$. There are now several alternative proofs of this result, notably [Pur13], [FK13], [Wes16] and [Wes19]. For an overview of some of these approaches, see [Rhe12].

It turns out that it is possible to determine whether a polynomial reduces modulo $q^{n}-1$ to the character of a cyclic group action of order $n$.

Theorem 3 ([AA19, Thm. 2.7]). Set $f(q) \in \mathbb{N}_{0}[q]$ and suppose $f\left(\xi^{d}\right) \in \mathbb{N}_{0}$ for all $d \in\{1, \ldots, n\}$, where $\xi$ is a primitive $n$th root of unity. Let $X$ be any set of size $f(1)$.

Then there exists a cyclic group action $\rho$ of order $n$ such that $(X,\langle\rho\rangle, f(q))$ exhibits the cyclic sieving phenomenon if and only if for every $k \mid n$,

$$
\sum_{d \mid k} \mu(k / d) f\left(\xi^{d}\right) \geq 0
$$

where $\mu$ is the number-theoretic Möbius function.

Remark 4. Except for its size, the nature of the set $X$ is irrelevant in Theorem 3.

Remark 5. If $(X,\langle\rho\rangle, f(q))$ exhibits the cyclic sieving phenomenon, the expression

$$
\frac{1}{k} \sum_{d \mid k} \mu(k / d) f\left(\xi^{d}\right)
$$

is the number of orbits of size $k$ of the group action. Therefore, the sum

$$
\sum_{d \mid k} \mu(k / d) f\left(\xi^{d}\right)
$$

must be nonnegative and divisible by $k$. The condition that the sum be divisible by $k$ follows from the hypothesis that $f(q) \in \mathbb{N}_{0}[q]$ and $f\left(\xi^{d}\right) \in \mathbb{N}_{0}$ for all $d \in\{1, \ldots, n\}$ (see [AA19, Lem. 2.5]).

Remark 6. It may be the case that $f(q) \in \mathbb{N}_{0}[q]$ evaluates to nonnegative integers at $n$th roots of unity but is not a cyclic sieving polynomial. As an example (see [AA19, Ex. 2.10]), take $f(q)=$ $q^{5}+3 q^{3}+q+10$. At 6 th roots of unity, $f\left(\xi^{j}\right)$ takes nonnegative integer values. However, for $k=3$ we have $\sum_{d \mid k} \mu(k / d) f\left(\xi^{d}\right)=-3$.

We conclude this section by recalling a fact that makes cyclic groups special. In general, two nonisomorphic group actions may have the same linear character. This is not the case for group actions of a cyclic group, as Brauer's permutation lemma shows:

Theorem 7 ([Bra41, Kov82]). Two cyclic group actions are isomorphic if and only if they are isomorphic as linear representation - that is, their characters coincide. 


\section{Some properties of skew Schur functions}

In this section we recall some basic properties of skew Schur functions, the Littlewood-Richardson coefficients and fake-degree polynomials. We refer to the books by Macdonald [Mac95] and Stanley [Sta01] for definitions. We use English notation for tableaux in all our figures.

Let $\operatorname{SYT}(\lambda / \mu)$ and $\operatorname{SSYT}(\lambda / \mu)$ be the set of standard and semistandard Young tableaux of skew shape $\lambda / \mu$, respectively. Given a skew shape $\lambda / \mu$ with $n$ cells, the associated skew Schur function $\mathrm{s}_{\lambda / \mu}$ is defined as

$$
\mathrm{s}_{\lambda / \mu}(\mathbf{x}):=\sum_{T \in \operatorname{SSYT}(\lambda / \mu)} \prod_{\square \in \lambda / \mu} x_{T(\square)} .
$$

This generalises the ordinary Schur function $s_{\lambda}:=s_{\lambda / \emptyset}$. It is well known that $\left\{s_{\lambda}\right\}_{\lambda}$, where $\lambda$ runs over all partitions, is a basis for the ring of symmetric functions. Another basis is given by the set of power-sum symmetric functions indexed by partitions. These are defined as

$$
\mathrm{p}_{v}(\mathbf{x}):=\mathrm{p}_{v_{1}}(\mathbf{x}) \mathrm{p}_{v_{2}}(\mathbf{x}) \cdots \mathrm{p}_{v_{\ell}}(\mathbf{x}), \quad \mathrm{p}_{j}(\mathbf{x}):=x_{1}^{j}+x_{2}^{j}+\cdots
$$

The skew characters $\chi^{\lambda / \mu}(v)$ of the symmetric group $\mathfrak{S}_{n}$ are then defined implicitly via

$$
\mathrm{s}_{\lambda / \mu}(\mathbf{x})=\sum_{v} \chi^{\lambda / \mu}(v) \frac{\mathrm{p}_{v}(\mathbf{x})}{z_{v}},
$$

where the sum is over all partitions $v$ of the same size as $\lambda / \mu, z_{\nu}=\prod_{j} m_{j} ! j^{m_{j}}$ and $m_{j}$ is the number of parts in $\lambda$ equal to $j$.

We define the Littlewood-Richardson coefficients $c_{\mu \nu}^{\lambda} \in \mathbb{N}_{0}$ via the expansion of the skew Schur function $\mathrm{s}_{\lambda / \mu}$ in the basis of the ordinary Schur functions,

$$
\mathrm{s}_{\lambda / \mu}(\mathbf{x})=\sum_{\nu} c_{\mu, \nu}^{\lambda} \mathrm{s}_{v}(\mathbf{x})
$$

Note that $c_{\mu \nu}^{\lambda}=0$ if $\nu$ and $\lambda / \mu$ are not of the same size. Combining equations (1) and (2), we obtain an equivalent expansion for the skew characters,

$$
\chi^{\lambda / \mu}=\sum_{\nu} c_{\mu, \nu}^{\lambda} \chi^{\nu}
$$

Although it is very difficult to determine whether a given Littlewood-Richardson coefficient vanishes, the following particular case is straightforward.

Lemma 8. Let $\lambda / \mu$ be a skew shape with $n$ cells. Then $c_{\mu,(n)}^{\lambda}=0$ if and only if $\lambda / \mu$ has a column with at least two cells. Similarly, $c_{\mu,\left(1^{n}\right)}^{\lambda}=0$ if and only if $\lambda / \mu$ has a row with at least two cells.

Proof. We shall first prove the statement

$$
c_{\mu,(n)}^{\lambda}=0 \Longleftrightarrow \lambda / \mu \text { has a column with at least two cells. }
$$

We expand both sides of equation (2) in the monomial basis. The left-hand side contains the monomial $x_{1}^{n}$ if and only if there is no column of $\lambda / \mu$ with at least two cells. Since the only semistandard Young tableau of straight shape that contains precisely $n$ times the letter 1 has shape $(n)$, the monomial $x_{1}^{n}$ appears in the right-hand side if and only if $c_{\mu,(n)}^{\lambda} \neq 0$. 
The statement concerning $c_{\mu,\left(1^{n}\right)}^{\lambda}$ follows by applying the involution $\omega$, with the property that $\omega\left(s_{\lambda / \mu}\right)=s_{\lambda^{\prime} / \mu^{\prime}}$, on both sides of equation (2). This yields

$$
\mathrm{s}_{\lambda^{\prime} / \mu^{\prime}}(\mathbf{x})=\sum_{v} c_{\mu, \nu}^{\lambda} \mathrm{s}_{\nu^{\prime}}(\mathbf{x})
$$

A similar argument as in the previous paragraph now finishes the proof.

Definition 9. Given a skew standard Young tableau $T$ with $n$ cells, a label $j$ with $1 \leq j<n$ is a descent of $T$ if the label $j+1$ appears in a row strictly below that of $j$. The major index of $T$, denoted $\operatorname{maj}(T)$, is the sum of the descents of $T$. The fake-degree polynomial $f^{\lambda / \mu}(q)$ associated with a skew Young diagram $\lambda / \mu$ is the generating function for the major index:

$$
f^{\lambda / \mu}(q):=\sum_{T \in \operatorname{SYT}(\lambda / \mu)} q^{\operatorname{maj}(T)} .
$$

The following lemma relates skew Schur functions and fake-degree polynomials:

Lemma 10 ([Sta01, Prop. 7.19.11]). Let $\lambda / \mu$ be a skew shape with $n$ cells. Then

$$
\mathrm{s}_{\lambda / \mu}\left(1, q, q^{2}, \ldots\right)=\frac{f^{\lambda / \mu}(q)}{(1-q)\left(1-q^{2}\right) \cdots\left(1-q^{n}\right)} .
$$

\section{Skew characters and their fake degrees}

A result by Springer [Spr74] gives an expression for the evaluation of an irreducible character of the symmetric group at a power of the long cycle $(1, \ldots, n) \in \mathfrak{S}_{n}$. In this section, we extend this result to skew characters.

Proposition 11. Let $\lambda / \mu$ be a skew shape with $n$ cells and let $\xi$ be a primitive $n$th root of unity. Then, for $n=d m$,

$$
\chi^{\lambda / \mu}\left(\left(m^{d}\right)\right)=f^{\lambda / \mu}\left(\xi^{d}\right)
$$

We shall deduce this from the following, more general result. This was first proved explicitly by Sagan, Shareshian and Wachs [SSW11, Prop. 3.1]. As they note, however, it is already implicit in work of Désarménien [Dés83]. We include yet another, different, proof.

Proposition 12. Let $F(\mathbf{x})$ be a homogeneous symmetric function of degree $n$, such that

$$
F(\mathbf{x})=\sum_{v \vdash n} \chi^{F}(v) \frac{\mathrm{p}_{v}(\mathbf{x})}{z_{v}} .
$$

Furthermore, let $f^{F}(q)$ be the following variation of the principal specialisation of $F(\mathbf{x})$ :

$$
f^{F}(q):=\left(\prod_{j=1}^{n}\left(1-q^{j}\right)\right) F\left(1, q, q^{2}, \ldots\right) .
$$

Then, for a primitive nth root of unity $\xi$ and $n=d m$, we have $\chi^{F}\left(\left(m^{d}\right)\right)=f^{F}\left(\xi^{d}\right)$.

Proof. Substituting $\mathrm{p}_{k}\left(1, q, q^{2}, \ldots\right)=\left(1-q^{k}\right)^{-1}$ in $F(\mathbf{x})$, we obtain

$$
f^{F}(q)=\left(\prod_{j=1}^{n}\left(1-q^{j}\right)\right) \sum_{\nu \vdash n} \frac{\chi^{F}(v)}{z_{v}} \prod_{k=1}^{\ell(\nu)} \frac{1}{1-q^{v_{k}}} .
$$


Each summand on the right-hand side approaches 0 as $q \rightarrow \xi^{d}$, unless $v=\left(m^{d}\right)$, because the first product has a zero with multiplicity $d$ at $q=\xi^{d}$. Taking the limit, removing all summands other than the one corresponding to $v=\left(m^{d}\right)$ and rearranging the expression slightly, we find

$$
\lim _{q \rightarrow \xi^{d}} f^{F}(q)=\lim _{q \rightarrow \xi^{d}}\left(\prod_{k=1}^{d} \frac{1}{1-q^{k m}} \prod_{j=1}^{n}\left(1-q^{j}\right)\right) \frac{\chi^{F}\left(\left(m^{d}\right)\right)}{z_{\left(m^{d}\right)}} \prod_{k=1}^{d} \frac{1-q^{k m}}{1-q^{m}} .
$$

The last product approaches $d$ ! by L'Hospital's rule, and $z_{\left(m^{d}\right)}=d ! m^{d}$. Since $n=d m$, the first two products are expressible as

$$
\prod_{k=1}^{d} \frac{1}{1-q^{k m}} \prod_{j=1}^{n}\left(1-q^{j}\right)=\prod_{k=0}^{d-1} \prod_{j=1}^{m-1}\left(1-q^{j+k m}\right) .
$$

Now, since $\xi^{m d}=1$, we obtain

$$
f^{F}\left(\xi^{d}\right)=\left(\prod_{j=1}^{m-1}\left(1-\xi^{j d}\right)\right)^{d} \frac{\chi^{F}\left(\left(m^{d}\right)\right)}{m^{d}}=\left(\frac{1}{m} \prod_{j=1}^{m-1}\left(1-\xi^{j d}\right)\right)^{d} \chi^{F}\left(\left(m^{d}\right)\right) .
$$

Because $\xi^{d}$ is a primitive $m$ th root of unity, $x^{m}-1=\prod_{j=0}^{m-1}\left(x-\xi^{j d}\right)$. Dividing both sides by $x-1$ gives the identity

$$
\left(x^{m-1}+x^{m-2}+\cdots+x+1\right)=\prod_{j=1}^{m-1}\left(x-\xi^{j d}\right) .
$$

Setting $x=1$ here shows that $\prod_{j=1}^{m-1}\left(1-\xi^{j d}\right)=m$, and this final observation shows that the right-hand side of equation (4) equals $\chi^{F}\left(\left(m^{d}\right)\right)$.

Proof of Proposition 11. By Lemma 10, this is the special case of Proposition 12 where $F(\mathbf{x})=$ $\mathrm{s}_{\lambda / \mu}(\mathbf{x})$.

\section{Skew border-strip tableaux and the abacus}

In this section, we recall the definition of border-strip tableaux, abaci and the Littlewood map. We end with a generalisation of a theorem of James and Kerber.

A border strip (or ribbon or skew hook) is a connected nonempty skew Young diagram containing no $2 \times 2$-square of cells, as in Figure 1 . The height of a border strip is one less than the number of rows it spans.

Let $\lambda / \mu$ be a skew shape. The size of $\lambda / \mu$ is its number of cells, denoted $|\lambda / \mu|$. Suppose that $v=\left(v_{1}, \ldots, v_{\ell}\right)$ is a partition of $|\lambda / \mu|$. A border-strip tableau of shape $\lambda / \mu$ and type $v$ is a tiling of the Young diagram of $\lambda / \mu$ with labelled border strips $B_{1}, \ldots, B_{\ell}$ with the following properties:

$\circ$ the border strip $B_{j}$ has label $j$ and size $v_{j}$, and

$\circ$ labelling all cells in $B_{j}$ with $j$ results in a labelling of the diagram $\lambda / \mu$ where labels in every row and every column are weakly increasing.

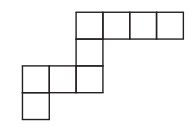

Figure 1. A border-strip of height 3. 

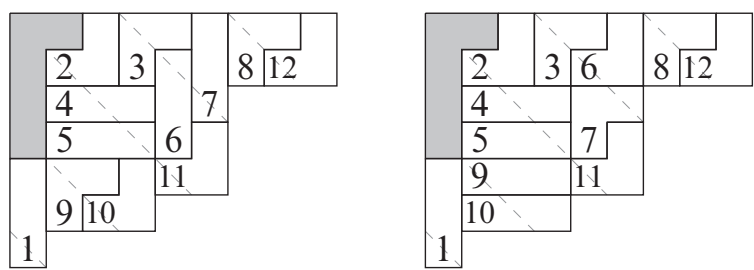

Figure 2. Two border-strip tableaux in BST $\left(\left(9^{2}, 6^{3}, 4,1\right) /\left(2,1^{3}\right), 3\right)$ of heights 13 and 9 , respectively. In each strip, the label has been placed in the cell with minimal content.

We let $\operatorname{BST}(\lambda / \mu, \nu)$ denote the set of all such border-strip tableaux. In particular, $\operatorname{BST}\left(\lambda / \mu, 1^{n}\right)$ may be identified with the set of standard Young tableaux of shape $\lambda / \mu$. In the remainder of the paper, we shall only concern ourselves with border-strip tableaux where all strips have the same size $d$, which we denote by $\operatorname{BST}(\lambda / \mu, d)$.

The height of a border-strip tableau $T$, or any tiling of a tableau with border strips, is the sum of the heights of the border strips in the partition. The content of a cell is given by its column index minus its row index. Observe that within a border strip, the lowest leftmost cell has the smallest content. By convention, the label of a strip is placed in the unique cell with minimal content in the strip, as shown in Figure 2.

In equation (1), the skew characters $\chi^{\lambda / \mu}$ were defined. The skew Murnaghan-Nakayama rule describes a way to compute these skew characters.

Theorem 13 (Murnaghan-Nakayama; see [Sta01, Cor. 7.17.5]). The skew characters are given by the signed sum

$$
\chi^{\lambda / \mu}(v)=\sum_{B \in \operatorname{BST}(\lambda / \mu, \nu)}(-1)^{\operatorname{height}(B)} .
$$

We will use the abacus model introduced by James and Kerber [JK84, Ch. 2.7] to encode partitions and their quotients.

Definition 14. A bead sequence is an infinite binary word $w=\left(w_{i}\right)_{i \in \mathbb{N} \geq 1}$ such that only a finite number of letters, the beads, are equal to 1 .

Recording for each 1 in $w$ the total number of 0s preceding it yields, apart from some leading zeros, the sequence of parts of an integer partition $\lambda$, ordered from the smallest part to the largest. In this case, $w$ is a bead sequence for $\lambda$.

The $d$-abacus on $w$ is the $d$-tuple of runners $\left(w^{1}, \ldots, w^{d}\right)$, with $w^{s}=\left(w_{i d+s}\right)_{i \in \mathbb{N}_{0}}$ for $1 \leq s \leq d$.

The partition corresponding to a bead sequence can alternatively be obtained by interpreting each 0 as a horizontal unit step and each 1 as a vertical unit step. This yields, apart from some leading vertical steps, the path tracing the southeast border of the Young diagram, in English notation.

Thus, prepending a 1 to a bead sequence, we obtain another bead sequence for the same partition. The $d$-abacus on the modified bead sequence is obtained from the $d$-abacus on the original bead sequence by prepending a bead to the $d$ th runner and then cyclically shifting the runners.

We visualise a $d$-abacus, as in the right of Figure 3, by drawing $d$ runners as vertical lines. Each runner consists of equally spaced spots. Beginning with the top row and processing each row from left to right, a bead is placed if and only if the corresponding letter of the binary word is 1 . It will be convenient to colour beads before the first empty spot black, and label the remaining positions beginning with 0 . The labels of the beads are then the hook lengths of the cells in the first column of the associated partition.

We can now define the core and the quotient of a partition.

Definition 15. Let $\lambda$ be a partition and let $d \geq 1$ be an integer. Then the $d$-core of $\lambda$ is the partition associated with the $d$-abacus which is obtained from the $d$-abacus on any bead sequence for $\lambda$ by moving all beads up as far as possible. 

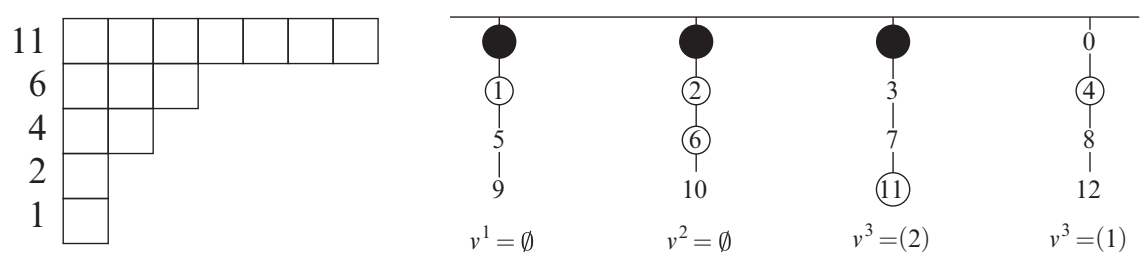

Figure 3. The 4-abacus $1110110101000010 \ldots$ for the partition $(7,3,2,1,1)$, together with its 4quotient.

Given a bead sequence $w$ for $\lambda$, the $d$-quotient of $\lambda$ is the $d$-tuple of partitions obtained by regarding each runner of the $d$-abacus on $w$ as a bead sequence.

Strictly speaking, the $d$-quotient of $\lambda$ depends on the chosen bead sequence - or more precisely, on its number of beads modulo $d$. However, as indicated before, choosing a different bead sequence corresponds to a cyclic permutation of the $d$-tuple of partitions in the $d$-quotient. Therefore, if the ordering of the partitions in the quotient is irrelevant, it is not necessary to explicitly fix a bead sequence.

By contrast, the $d$-core is independent of the chosen bead sequence. For example, the 4-core of the partition $(7,3,2,1,1)$ is $(2)$, as can be seen by moving the beads in positions 4 and 11 up in Figure 3 , obtaining the bead sequence $111111100100 \ldots$

The following observation is fundamental to the utility of the abacus.

Lemma 16. Suppose that the partition $\lambda$ is obtained from the partition $\mu$ by adding a border strip of size $d$, whose cell of minimal content has content $c$ in $\lambda$.

Then a $d$-abacus for $\mu$ is obtained from a $d$-abacus for $\lambda$ by moving a bead on the $j$ th runner up by one position. Provided that $d$ divides the total number of beads on the abacus, we have $j \equiv c(\bmod d)$.

Proof. Let $\left(w_{i}^{\lambda}\right)_{i \in \mathbb{N} \geq 1}$ be a bead sequence for $\lambda$, and let $x$ and $y$ be the column and the row, respectively, of the cell of minimal content of the border strip which was added to $\mu$ to obtain $\lambda$.

Let $j$ be the index of the $x$ th zero in $\left(w_{i}^{\lambda}\right)_{i \in \mathbb{N}_{\geq 1}}$. Thus, $w_{j}^{\lambda}, w_{j+1}^{\lambda}, \ldots, w_{j+d}^{\lambda}$ encodes the path tracing the southeast border of the Young diagram of $\lambda / \mu$. In particular, since $\lambda / \mu$ is a border strip, $w_{j+d}^{\lambda}=1$. Set

$$
w_{i}^{\mu}= \begin{cases}1 & \text { if } i=j \\ 0 & \text { if } i=j+d \\ w_{i}^{\lambda} & \text { otherwise }\end{cases}
$$

Equivalently, this is the bead sequence for the $d$-abacus obtained by moving a bead up on the $\bar{j}$ th runner, with $\bar{j} \equiv j(\bmod d)$. Moreover, $w_{j}^{\mu}, w_{j+1}^{\mu}, \ldots, w_{j+d}^{\mu}$ encodes the path tracing the northwest border of the Young diagram of $\lambda / \mu$, so $\left(w_{i}^{\mu}\right)_{i \in \mathbb{N}_{\geq 1}}$ is indeed a bead sequence for $\mu$.

Let $\ell$ be the total number of $1 \mathrm{~s}$ in $\left(w_{i}^{\lambda}\right)_{i \in \mathbb{N}_{\geq 1}}$. Then there are $x-10 \mathrm{~s}$ in $w_{1}^{\lambda}, \ldots, w_{j-1}^{\lambda}$ and $\ell-y 1 \mathrm{~s}$. Thus, provided that $\ell=d k$, we have $j=x-1+d k-y+1 \equiv x-y(\bmod d)$.

Definition 17. Let $\lambda / \mu$ be a skew shape. Then $\mathbf{w}=\left(w_{i}^{\lambda}, w_{i}^{\mu}\right)_{i \in \mathbb{N}_{\geq 1}}$ is a (skew) bead sequence for $\lambda / \mu$ if $\left(w_{i}^{\lambda}\right)_{i \in \mathbb{N} \geq 1}$ and $\left(w_{i}^{\mu}\right)_{i \in \mathbb{N}_{\geq 1}}$ are bead sequences for $\lambda$ and $\mu$, respectively, and the number of beads in these two bead sequences are the same.

The (skew) $d$-abacus on $\mathbf{w}$ is the pair of $d$-abaci for $\left(w_{i}^{\lambda}\right)_{i \in \mathbb{N}_{\geq 1}}$ and $\left(w_{i}^{\mu}\right)_{i \in \mathbb{N}_{\geq 1}}$.

Suppose that there is a (skew) bead sequence $\mathbf{w}$ for $\lambda / \mu$ such that for all $1 \leq i \leq d$, the $i$ th pair of runners in the $d$-abacus on $\mathbf{w}$ is a bead sequence for a skew shape $v^{i} / \kappa^{i}$. Then the corresponding $d$-quotient of $\lambda / \mu$ is the $d$-tuple $\left(v^{1} / \kappa^{1}, \ldots, v^{d} / \kappa^{d}\right)$. If there is no such bead sequence, the $d$-quotient of $\lambda / \mu$ does not exist. 

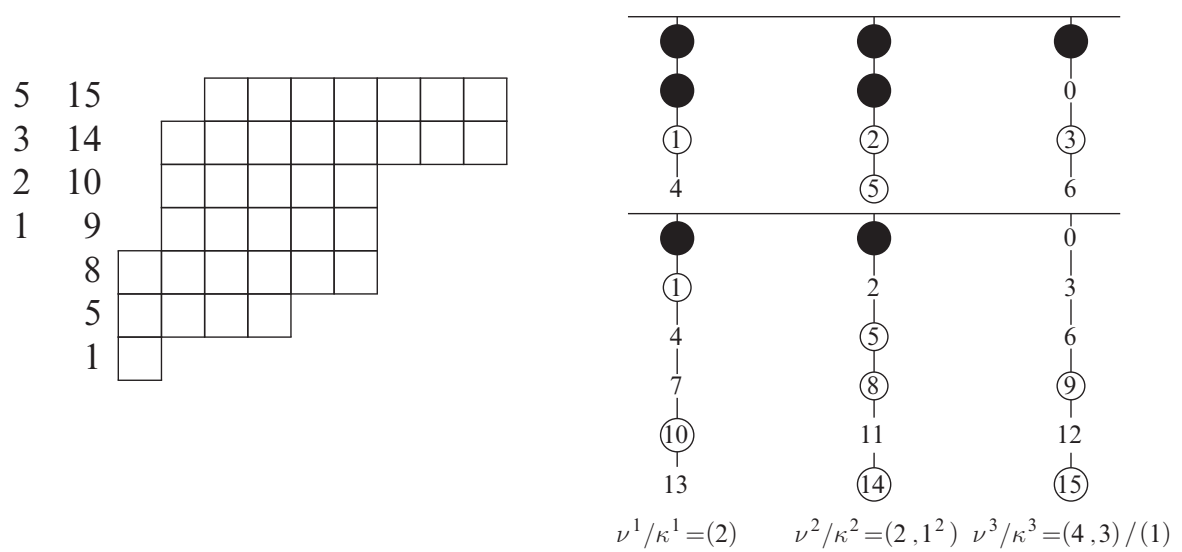

Figure 4. The hook lengths of the first column of the inner and the outer shape of the diagram in Figure 2, and the pair of 3-abaci corresponding to the shape.

We visualise a $d$-abacus for $\lambda / \mu$ by drawing the $d$-abacus for $\mu$ above the $d$-abacus for $\lambda$, as on the right-hand side of Figure 4.

Lemma 18. Let $\lambda / \mu$ be a skew shape. Then the $d$-quotient of $\lambda / \mu$ exists if and only if $\operatorname{BST}(\lambda / \mu, d)$ is nonempty.

Proof. It is an immediate consequence of Lemma 16 that $\operatorname{BST}(\lambda / \mu, d)$ is nonempty if and only if a $d$-abacus for $\mu$ can be obtained from the $d$-abacus for $\lambda$ by moving beads up along their runners. Put differently, each pair of runners in a $d$-abacus for $\lambda / \mu$ is a bead sequence for some skew shape.

Remark 19. Note that in this case the $d$-cores of $\lambda$ and $\mu$ coincide. In fact, a $d$-quotient of a partition $\lambda$ with core $\mu$ is precisely a $d$-quotient of $\lambda / \mu$.

We now recall the Littlewood map as described, for example, by James and Kerber [JK84, Ch. 2.7] or Pak [Pak00, fig. 2.6], where it is called the rim hook bijection.

Suppose that $\operatorname{BST}(\lambda / \mu, d)$ is nonempty. Then, given a bead sequence for $\lambda / \mu$, let the set of standard Young tableaux tuples, denoted by $\operatorname{SYTT}(\lambda / \mu, d)$, be the set of $d$-tuples $\left(T^{1}, T^{2}, \ldots, T^{d}\right)$ of skew tableaux with the following properties:

$\circ$ the shape of $T^{j}$ is $\lambda^{j} / \mu^{j}$, the $j$ th entry of the $d$-quotient of $\lambda / \mu$;

$\circ$ for each tableau $T^{j}$, the cell labels in rows and columns increase; and

$\circ$ each of the numbers $\{1,2, \ldots, n / d\}$ appears in precisely one tableau.

Having fixed a bead sequence for $\lambda / \mu$, the Littlewood map is a bijection between the sets $\operatorname{BST}(\lambda / \mu, d)$ and $\operatorname{SYTT}(\lambda / \mu, d)$. Let $\left(\lambda^{1} / \mu^{1}, \ldots, \lambda^{d} / \mu^{d}\right)$ be the $d$-quotient of $\lambda / \mu$ corresponding to the bead sequence. Set $B \in \operatorname{BST}(\lambda / \mu, d)$.

We define $\left(T^{1}, T^{2}, \ldots, T^{d}\right)$ inductively: if $\lambda / \mu$ is the empty partition, then $T^{i}$ is the empty partition for $1 \leq i \leq d$. Otherwise, let $\bar{B}$ be the border-strip tableau obtained from $B$ by deleting the strip with the largest label $n / d$, and let $\bar{\lambda} / \mu$ be its shape. Let $\left(\bar{\lambda}^{1} / \mu^{1}, \ldots, \bar{\lambda}^{d} / \mu^{d}\right)$ be the $d$-quotient of $\bar{\lambda} / \mu$ corresponding to the bead sequence which has as many beads as the chosen bead sequence for $\lambda / \mu$. Let $\left(\bar{T}^{1}, \bar{T}^{2}, \ldots, \bar{T}^{d}\right)$ be the standard Young tableau tuple corresponding to $\bar{B}$.

Then there is a unique index $1 \leq j \leq d$ with $\bar{\lambda}^{j} / \mu^{j} \neq \lambda^{j} / \mu^{j}$. Moreover, $\lambda^{j} / \mu^{j}$ is obtained from $\bar{\lambda}^{j} / \mu^{j}$ by adding a single cell. The standard Young tableaux tuple $\left(T^{1}, T^{2}, \ldots, T^{d}\right)$ is thus obtained from $\left(\bar{T}^{1}, \bar{T}^{2}, \ldots, \bar{T}^{d}\right)$ by placing $n / d$ into this cell of $T^{j}$.

Remark 20. Suppose that the number of beads in the chosen bead sequence for $\lambda / \mu$ is divisible by $d$. Let $c(x)$ denote the content of the unique cell with minimal content in the $d$-strip labelled $x$ in $B$, as shown in Figure 2. 
Then it follows immediately from Lemma 16 that any label $x$ appearing in $T^{j}$ satisfies $c \equiv j(\bmod d)$.

Furthermore, two labelled cells in $T^{j}$ differ in content by $k$ if and only if the two cells with these labels in $B$ differ in content by $d k$.

The images of the two border-strip tableaux in Figure 2 are given by the triples in expressions (5) and (6), respectively.

Example 21. The 3 -cores of $\lambda=\left(9^{2}, 6^{3}, 4,1\right)$ and $\mu=\left(2,1^{3}\right)$ are both given by the partition (2). Provided we choose bead sequences such that the number of beads is divisible by 3 , the 3quotient of $\lambda$ is $(2),\left(2,1^{2}\right),(4,3)$, and the 3 -quotient of $\mu$ is $\emptyset, \emptyset,(1)$. Thus, the 3 -quotient of $\lambda / \mu$ is $(2),\left(2,1^{2}\right),(4,3) /(1)$, and the two tuples

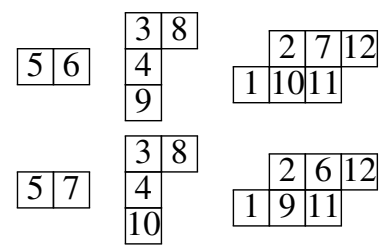

are elements in $\operatorname{SYTT}(\lambda / \mu, 3)$, corresponding to the two tableaux in Figure 2.

Theorem 22. Let $\lambda / \mu$ be a skew shape of size $n$ and let $k$ and $d$ be positive integers with $d k \mid n$. Fix a skew bead sequence for $\lambda / \mu$.

Then $\operatorname{BST}(\lambda / \mu, d k)$ is nonempty if and only if $\operatorname{BST}(\lambda / \mu, k)$ is nonempty and, for the $k$-quotient $\left(v^{1} / \kappa^{1}, \ldots, v^{k} / \kappa^{k}\right)$ of $\lambda / \mu$, each set $\mathrm{BST}\left(v^{i} / \kappa^{i}, d\right)$ is nonempty, for $1 \leq i \leq k$.

In this case, $d \mid \operatorname{gcd}\left(\left|v^{1} / \kappa^{1}\right|, \ldots,\left|v^{k} / \kappa^{k}\right|\right)$ and

$$
|\operatorname{BST}(\lambda / \mu, d k)|=\left(\begin{array}{c}
\sum_{i}\left|v^{i} / \kappa^{i}\right| / d \\
\left|v^{1} / \kappa^{1}\right| / d, \ldots,\left|v^{k} / \kappa^{k}\right| / d
\end{array}\right) \prod_{i=1}^{k}\left|\operatorname{BST}\left(v^{i} / \kappa^{i}, d\right)\right| .
$$

Remark 23. The case $d=1$ for straight shapes is classical and can be found in [JK84, eq. (2.7.32)] or [FL97].

Proof. Let us first prove the special case $d=1$. Since the Littlewood map is bijective, it suffices to count the number of standard Young tableaux tuples $\left(T^{1}, T^{2}, \ldots, T^{d}\right)$ with shapes $\left(v^{1} / \kappa^{1}, \ldots, v^{k} / \kappa^{k}\right)$. This number is

$$
|\operatorname{BST}(\lambda / \mu, k)|=|\operatorname{SYTT}(\lambda / \mu, k)|=\left(\begin{array}{c}
\sum_{i}\left|v^{i} / \kappa^{i}\right| \\
\left|v^{1} / \kappa^{1}\right|, \ldots,\left|v^{k} / \kappa^{k}\right|
\end{array}\right) \prod_{i=1}^{k}\left|\operatorname{SYT}\left(v^{i} / \kappa^{i}\right)\right|,
$$

where the multinomial coefficient counts the number of ways to distribute the labels among the skew shapes.

Now we reduce the general statement to the case $d=1$. First notice that $|\operatorname{BST}(\lambda / \mu, d k)| \leq$ $|\operatorname{BST}(\lambda / \mu, k)|$, because there exists a natural injection which splits each strip of length $d k$ into $d$ strips of length $k$.

Let $\mathbf{w}=\left(w_{i}^{\lambda}, w_{i}^{\mu}\right)_{i \in \mathbb{N}_{\geq 1}}$ be a bead sequence for $\lambda / \mu$. For simplicity, we write $\mathbf{w}_{i}:=\left(w_{i}^{\lambda}, w_{i}^{\mu}\right)$. For $1 \leq s \leq k$, let $\mathbf{w}^{s}=\left(\mathbf{w}_{i k+s}\right)_{i \in \mathbb{N}_{0}}$ be the $s$ th runner in the $k$-abacus on $\mathbf{w}$.

By Lemma 18 , it suffices to prove that all runners in the $d k$-abacus on $\mathbf{w}$ are bead sequences for skew shapes if and only if all runners in the $d$-abacus on $\mathbf{w}^{s}$ are bead sequences for skew shapes for all $1 \leq s \leq k$. For $1 \leq t \leq d$, the $t$ th runner in the $d$-abacus on $\mathbf{w}^{s}$ is

$$
\mathbf{w}^{s, t}=\left(\mathbf{w}_{(j \cdot d+t-1) \cdot k+s}\right)_{j \in \mathbb{N}_{0}}=\left(\mathbf{w}_{(j \cdot(d k)+(t-1) k+s}\right)_{j \in \mathbb{N}_{0}} .
$$


Because $1 \leq(t-1) k+s<d k$, this is precisely the $((t-1) k+s)$ th runner in the $d k$-abacus on $\mathbf{w}$, giving the equivalence.

Suppose now that $|\operatorname{BST}(\lambda / \mu, d k)|$ is nonempty and let

$$
\left(v^{s, 1} / \kappa^{s, 1}, \ldots, v^{s, d} / \kappa^{s, d}\right)
$$

be the $d$-quotient of $v^{s} / \kappa^{s}$. The earlier equation on runners shows that the collection of skew shapes $v^{s, t} / \kappa^{s, t}$ for $1 \leq s \leq k$ and $1 \leq t \leq d$ coincides with the collection of skew shapes in the $d k$-quotient for $\lambda / \mu$.

To conclude the argument, we compute

$$
\begin{aligned}
\prod_{i=1}^{k}\left|\operatorname{BST}\left(v^{i} / \kappa^{i}, d\right)\right| & =\prod_{i=1}^{k}\left(\begin{array}{c}
\left|v^{i} / \kappa^{i}\right| / d \\
\left.\left|v^{i, 1} / \kappa^{i, 1}\right|, \ldots,\left|v^{i, d} / \kappa^{i, d}\right|\right)
\end{array}\right) \prod_{j=1}^{d}\left|\operatorname{SYT}\left(v^{i, j} / \kappa^{i, j}\right)\right| \\
& =\prod_{i=1}^{k} \prod_{j=1}^{d}\left|\operatorname{SYT}\left(v^{i, j} / \kappa^{i, j}\right)\right|,
\end{aligned}
$$

and finally obtain

$$
\left(\begin{array}{c}
|\lambda / \mu| / d k \\
\left|v^{1} / \kappa^{1}\right| / d, \ldots,\left|v^{k} / \kappa^{k}\right| / d
\end{array}\right) \prod_{i=1}^{k}\left|\operatorname{BST}\left(v^{i} / \kappa^{i}, d\right)\right|=|\operatorname{BST}(\lambda / \mu, d k)| .
$$

Example 24. Consider the skew shape $\lambda / \mu=\left(9,7,4^{2}, 3^{2}, 1\right) /\left(3,2,1^{2}\right)$ of 24 . A corresponding skew 6 -abacus is as follows. Note that the lower abacus, corresponding to the outer shape $\lambda$, can be obtained from the upper abacus, corresponding to the inner shape $\mu$, by moving beads down along a runner. Following Lemma 16 , this corresponds to the fact that $\operatorname{BST}(\lambda / \mu, 6)$ is nonempty.

\begin{tabular}{cccccc}
\hline 0 & 1 & 1 & 0 & 1 & 1 \\
3 & 1 & 1 & 1 & 1 & 1 \\
\hline 0 & 1 & 5 & 6 & 7 & 8 \\
1 & 1 & 2 & 3 & 4 & 5 \\
6 & 7 & 1 & 1 & 1 & 1 \\
1 & 1 & 1 & 9 & 10 & 11 \\
$(12)$ & 13 & 14 & 11 & 1 & 1 \\
$(2)$ & 0 & $(1)$ & $(2) /(1)$ & 0 & 0
\end{tabular}

On the other hand, the skew 3-abacus with the same number of beads is the following:

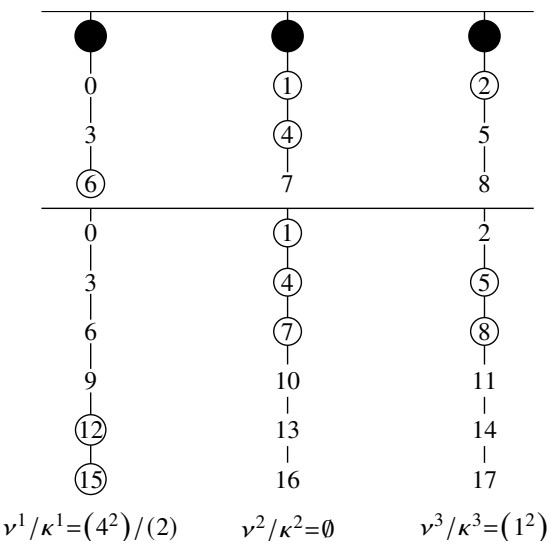


Finally, as an illustration of the proof, consider the skew 2-abacus for $v^{1} / \kappa^{1}$, corresponding to the first runner in the skew 3-abacus just shown and having the same number of beads. Observe that the (skew) bead sequences, and therefore also the corresponding skew shapes, are the same as on the first and the fourth runner of the 6-abacus:

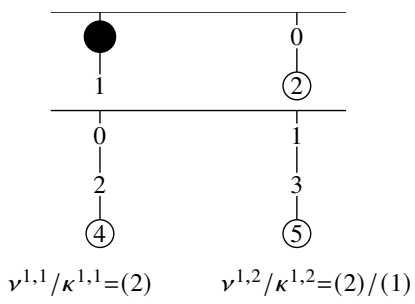

As a corollary, we obtain a useful characterisation of shapes with precisely one border-strip tableau.

Corollary 25. Let $\lambda / \mu$ be a skew shape of size $n \in \mathbb{N}_{\geq 1}$ and let $k$ be a positive integer with $k \mid n$. Suppose that $\operatorname{BST}(\lambda / \mu, k)$ is nonempty.

Then $\operatorname{BST}(\lambda / \mu, k)$ contains precisely one element if and only if all skew shapes in the skew $k$-quotient of $\lambda / \mu$ are empty, with exactly one exception, which is either a single row $(n / k)$ or a single column $\left(1^{n / k}\right)$.

In this case, $\lambda / \mu$ is a border strip and $\operatorname{BST}(\lambda / \mu, d k)$ contains precisely one element for all $d \mid \frac{n}{k}$.

Proof. Let $\left(v^{1} / \kappa^{1}, \ldots, v^{k} / \kappa^{k}\right)$ be the skew $k$-quotient of $\lambda / \mu$. Applying Theorem 22 with $d=1$, we obtain $|\operatorname{BST}(\lambda / \mu, k)|=1$ if and only if $\left|\operatorname{BST}\left(v^{i} / \kappa^{i}, 1\right)\right|=1$ for all $i$ and the multinomial coefficient in equation (7) evaluates to 1 .

Since BST $\left(v^{i} / \kappa^{i}, 1\right)$ is the set of standard Young tableaux of shape $v^{i} / \kappa^{i}$, we have that $\left|\operatorname{BST}\left(v^{i} / \kappa^{i}, 1\right)\right|=1$ if and only $v^{i} / \kappa^{i}$ is a single row or a single column. Furthermore, the multinomial coefficient equals 1 if $\left|v^{i} / \kappa^{i}\right|=0$ for all but one $i$.

Suppose now that $|\operatorname{BST}(\lambda / \mu, k)|=1$. Let $v^{j} / \kappa^{j}$ be the unique nonempty element in the quotient, which therefore has size $n / k$. Because $v^{j} / \kappa^{j}$ is a single row or a single column, $\left|\operatorname{BST}\left(v^{j} / \kappa^{j}, n / k\right)\right|=1$. For $i \neq j$ we trivially have $\left|\operatorname{BST}\left(v^{i} / \kappa^{i}, n / k\right)\right|=1$. Thus, by Theorem 22 with $d=n / k$, we have $|\operatorname{BST}(\lambda / \mu, n)|=\left|\operatorname{BST}\left(\lambda / \mu, \frac{n}{k} \cdot k\right)\right|=1$, which implies that $\lambda / \mu$ is a border strip.

Finally, recall that $|\operatorname{BST}(\lambda / \mu, d k)| \leq|\operatorname{BST}(\lambda / \mu, k)|$, because there is a natural injection which splits each strip of length $d k$ into $d$ strips of length $k$. Therefore, provided that $d \mid \frac{n}{k}$,

$$
1=|\operatorname{BST}(\lambda / \mu, n)| \leq|\operatorname{BST}(\lambda / \mu, d k)| \leq|\operatorname{BST}(\lambda / \mu, k)|=1 .
$$

\section{Skew characters and border-strip tableaux}

In this section we show that, up to sign, the evaluation of a skew character at a $d$ th power of a cycle equals the number of border-strip tableaux with all strips having size $d$. The nonskew case follows from a result by White [Whi83] and, via a different technique, by James and Kerber [JK84, Eq. 2.7.26]. Our proof is slightly different and uses techniques by Pak [Pak00]. The fact that the Murnaghan-Nakayama rule is cancellation-free in the skew case is also briefly stated in [LLT97, p.1047] and is implicit in [SSW03].

We include a straightforward proof here for convenience, as we have found no single reference which connects all three quantities of evaluations at roots of unity of fake-degree polynomials, skew characters and the number of skew border-strip tableaux.

Definition 26. Set $T=\left(T^{1}, \ldots, T^{d}\right) \in \operatorname{SYTT}(\lambda / \mu, d)$. Suppose that, after swapping labels $i$ and $i+1$ in $T$, the resulting tuple $T^{\prime}$ is still an element of $\operatorname{SYTT}(\lambda / \mu, d)$. Then this transposition is a flip on $T$.

The result of a flip on the border-strip tableau corresponding to $T$ under the Littlewood map is the image of $T^{\prime}$ under the Littlewood map. 
Example 27. The two flips $(6,7)$ and $(9,10)$ send the tableau tuple (5) to the tableau tuple (6). These correspond to the border-strip tableaux in Figure 2.

Lemma 28. All elements of $\operatorname{SYTT}(\lambda / \mu, d)$ are connected via a sequence of flips.

This lemma is essentially [Pak00, Thm. 3.2]. For convenience, we include a proof using our framework.

Proof. Fix a bead sequence for $\lambda / \mu$ and let $\left(\lambda^{1} / \mu^{1}, \ldots, \lambda^{d} / \mu^{d}\right)$ be the corresponding $d$-quotient of $\lambda / \mu$. Let us first describe the superstandard filling $S:=\left(S^{1}, \ldots, S^{d}\right) \in \operatorname{SYTT}(\lambda / \mu, d)$. We will then show that $S$ can be obtained from any other tableau by a sequence of flips.

The cells in $S^{1}$ are labelled with the numbers $1, \ldots,\left|\lambda^{1} / \mu^{1}\right|$, the cells in $S^{2}$ are labelled with numbers $\left|\lambda^{1} / \mu^{1}\right|+1, \ldots,\left|\lambda^{1} / \mu^{1}\right|+\left|\lambda^{2} / \mu^{2}\right|$ and so forth. The labels in each tableau $S^{i}$ are then distributed in the lexicographically smallest fashion, when reading row by row from top to bottom.

It now suffices to prove that for arbitrary $T \in \operatorname{SYTT}(\lambda / \mu, d)$, we can obtain $S$ from $T$ by a sequence of flips. We describe a sorting algorithm which rearranges the labels, starting with 1 and then continuing with $2,3, \ldots$, so that these labels agree with the corresponding labels in $S$.

Suppose that at some point during the procedure, all cells in $T$ with labels at most $i-1$ agree with $S$, but the cell labelled $i$ in $S$ is labelled $j>i$ in $T$. We then first flip $j$ and $j-1$, then $j-1$ and $j-2$, and so on, until $i+1$ and $i$ are flipped, at which point $i$ is in the correct spot. We show inductively that all these flips are possible to perform: by construction, the cells above and to the left of the cell labelled with $j$ in $T$ must have labels smaller than $i<j-1$. Because all tableaux in $T$ are standard, the cells below and to the right of the cell labelled with $j-1$ contain labels strictly larger than $j$. Performing this algorithm in order for all $i=1,2, \ldots$ ensures that we eventually reach $S$ from $T$.

By analysing the effect of flipping $i$ and $i+1$ on the corresponding border-strip tableau, we now show that the height of the latter remains invariant.

Lemma 29 ([Pak00, Lem. 4.1]). Suppose that $B$ and $B^{\prime}$ in $\operatorname{BST}(\lambda / \mu, d)$ are related by a flip. Then $(-1)^{\text {height }(B)}=(-1)^{\text {height }\left(B^{\prime}\right)}$.

Proof. Let $T$ and $T^{\prime}$ be the standard Young tableau tuples corresponding to $B$ and $B^{\prime}$, respectively, and let $T^{\prime}$ be obtained from $T$ by flipping $i$ and $i+1$.

Since the tuples of shapes of $T$ and $T^{\prime}$ restricted to the labels $\{1, \ldots, j\}$ coincide for $j \notin\{i, i+1\}$, the border-strip tableaux $B$ and $B^{\prime}$ differ only in the two border strips labelled $i$ and $i+1$.

If these are disconnected - that is, no cell in the first strip is horizontally or vertically adjacent to a cell in the second - only the labels $i$ and $i+1$ are interchanged in $B$ and $B^{\prime}$.

Otherwise, the two border strips must form a skew shape, since labels are increasing in every row and every column of a border-strip tableau. Because this skew shape is a union of two border strips, it can be partitioned into three parts: a (nonempty) middle part which consists of all pairs of cells $\left(b_{1}, b_{2}\right)$ whose contents are equal, a part to the left of this middle part and a part to the right of this middle part.

A flip necessarily fixes the left and right parts, while it swaps the cells in the middle part. Hence, the strips either both increase or both decrease in height by 1, as illustrated in the following example:
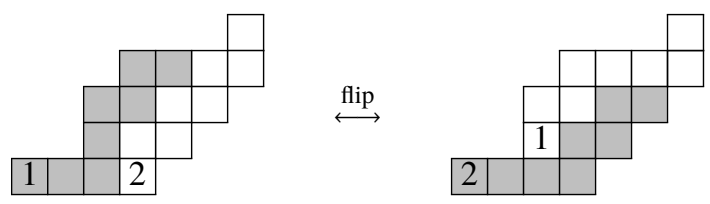

Together with Proposition 11, we can now conclude the main result of this section. 
Corollary 30. Let $\lambda / \mu$ be a skew shape of size $n=d m$. Then the signed sum

$$
\chi^{\lambda / \mu}\left(\left(m^{d}\right)\right)=\sum_{B \in \operatorname{BST}(\lambda / \mu, m)}(-1)^{\operatorname{height}(B)}
$$

in the Murnaghan-Nakayama rule (Theorem 13) is cancellation-free. In particular,

$$
f^{\lambda / \mu}\left(\xi^{d}\right)=\chi^{\lambda / \mu}\left(\left(m^{d}\right)\right)=\varepsilon|\operatorname{BST}(\lambda / \mu, m)|,
$$

where $\xi$ is a primitive $n$th root of unity and $\varepsilon=(-1)^{\text {height }(B)}$ for any $B \in \operatorname{BST}(\lambda / \mu, m)$.

\section{Bounds on the number of border-strip tableaux}

The goal of this section is to prove the following theorem:

Theorem 31. Let $\lambda / \mu$ be a skew shape with $n$ cells and let $k$ be a positive integer with $k \mid n$. Suppose that $|\operatorname{BST}(\lambda / \mu, k)| \geq 2$. Then

$$
|\operatorname{BST}(\lambda / \mu, k)| \geq \sum_{d \mid \frac{n}{k}, d>1}|\operatorname{BST}(\lambda / \mu, d k)| .
$$

Additionally, the inequality holds if $n / k$ is a prime number.

Example 32. For $\lambda=\left(10,1^{2}\right) \vdash 12$, we have $|\operatorname{BST}(\lambda, 3)|=1$ and $|\operatorname{BST}(\lambda, 6)|+|\operatorname{BST}(\lambda, 12)|=2$. By contrast, for $\lambda=\left(9,1^{3}\right) \vdash 12$, we have $|\operatorname{BST}(\lambda, 1)|=165,|\operatorname{BST}(\lambda, 2)|=5,|\operatorname{BST}(\lambda, 3)|=3$ and $|\operatorname{BST}(\lambda, 4)|=|\operatorname{BST}(\lambda, 6)|=|\operatorname{BST}(\lambda, 12)|=1$, and therefore

$$
|\operatorname{BST}(\lambda, k)| \geq \sum_{d \mid \frac{n}{k}, d>1}|\operatorname{BST}(\lambda, d k)|
$$

for all $k$.

Remark 33. For $k=1$, apart from the single-row and single-column partitions, there are only three shapes $\lambda / \mu$ where equality is attained: $\left(2^{2}\right),\left(3^{2}\right)$ and $\left(2^{3}\right)$. Other than that, the minimal difference between the two sides of the inequality is attained for hooks of the form $(n-1,1)$. In this case it equals $n-\tau(n)$, where $\tau(n)$ is the number of divisors of $n$.

Our strategy is to reduce the theorem to the case of straight shapes and $k=1$, which we prove in Section 7.2, using a bound from Fomin and Lulov.

In Section 7.3 we extend this to the case of skew shapes and $k=1$, essentially using the expansion of a skew character into irreducible characters.

Finally, in Section 7.4 we deduce the general case from the inequality with $k=1$, using a bound on the quotient of a multinomial coefficient and a multinomial coefficient with stretched entries proved in Section 7.1, Theorem 22 and Corollary 25.

\subsection{Bounds on multinomial coefficients}

We first prove two inequalities related to multinomial coefficients. For this we use the approximation by Robbins.

Proposition 34 ([Rob55]). For any positive integer $n$,

$$
n !=\sqrt{2 \pi} n^{n+1 / 2} e^{-n+r_{n}} \quad \text { for some } \quad \frac{1}{12 n+1}<r_{n}<\frac{1}{12 n} .
$$


Lemma 35. For any positive integer $d$ and positive integers $m_{1}, \ldots, m_{k}$ summing to $m$,

$$
\frac{\left(\begin{array}{c}
d m \\
d m_{1}, \ldots, d m_{k}
\end{array}\right)}{\left(\begin{array}{c}
m \\
m_{1}, \ldots, m_{k}
\end{array}\right)} \geq \frac{1}{d^{(k-1) / 2}}\left(\frac{m^{m}}{\prod_{j=1}^{k} m_{j}^{m_{j}}}\right)^{d-1} .
$$

Proof. For $d=1$ or $k=1$ the statement is trivial, so we assume $d>1$ and $k>1$.

The left-hand side of formula (8) is equal to

$$
\frac{(d m) !}{\prod_{j}\left(d m_{j}\right) !} \frac{\prod_{j}\left(m_{j}\right) !}{m !}
$$

which by Proposition 34 is larger than or equal to

$$
\frac{m^{(d-1) m}}{d^{(k-1) / 2} \prod_{i} m_{i}^{(d-1) m_{i}}} \exp \left(\frac{1}{12 d m+1}-\frac{1}{12 m}+\sum_{j} \frac{1}{12 m_{j}+1}-\frac{1}{12 d m_{j}}\right) .
$$

It remains to show that for $\varepsilon=1 / 12$,

$$
\frac{1}{d m+\varepsilon}+\sum_{j} \frac{1}{m_{j}+\varepsilon} \geq \frac{1}{m}+\sum_{j} \frac{1}{d m_{j}}
$$

provided $k>1$ and $d>1$. Set $M=m \sum_{j} \frac{1}{m_{j}}$ and note that $M \geq k \geq 2$. Furthermore, $m \sum_{j} \frac{1}{m_{j}+\varepsilon} \geq \frac{m}{1+\varepsilon} \sum_{j} \frac{1}{m_{j}}=\frac{1}{1+\varepsilon} M$ and $\frac{d m}{d m+\varepsilon} \geq \frac{1}{1+\varepsilon}$. Thus it suffices to prove that for $d \geq 2, M \geq 2$ and $\varepsilon=1 / 12$,

$$
\frac{1}{1+\varepsilon}(1+d M) \geq d+M
$$

This can be seen, for example, by replacing $d$ with $2+\tilde{d}$ and $M$ with $2+\tilde{M}$.

Corollary 36. For any integer $d>1$ and $k>1$ positive integers $m_{1}, \ldots, m_{k}$ summing to $m$,

$$
\frac{\left(\begin{array}{c}
d m \\
d m_{1}, \ldots, d m_{k}
\end{array}\right)}{\left(\begin{array}{c}
m \\
m_{1}, \ldots, m_{k}
\end{array}\right)} \geq \prod_{j=1}^{k}\left(\tau\left(d m_{j}\right)-1\right)
$$

where $\tau(n)$ is the number of divisors of $n$.

Proof. We use the inequality $n / 2 \geq \tau(n)-1$, valid for $n \geq 1$. By Lemma 35, it is then sufficient to show that

$$
\frac{1}{d^{(k-1) / 2}}\left(\frac{m^{m}}{\prod_{j=1}^{k} m_{j}^{m_{j}}}\right)^{d-1}>\left(\frac{d}{2}\right)^{k} \prod_{j=1}^{k} m_{j}
$$

or equivalently,

$$
\left(\prod_{j=1}^{k} \frac{1}{m_{j}}\right)\left(\frac{m^{m}}{\prod_{j=1}^{k} m_{j}^{m_{j}}}\right)^{d-1}>\frac{1}{\sqrt{d}}\left(\frac{d}{2}\right)^{k} d^{k / 2} .
$$


We will show the stronger inequality

$$
\left(\frac{m^{m}}{\prod_{j=1}^{k} m_{j}^{m_{j}+1}}\right)^{\frac{1}{k}}>\left(\frac{d \sqrt{d}}{2}\right)^{\frac{1}{d-1}}
$$

It is not hard to see that the right-hand side of this inequality, as a real function of $d>1$, attains its maximum between 3 and 4 and is unimodal. By direct inspection we see that for integral $d$, the maximum of the right-hand side is attained at $d=3$, where it is $\left(\frac{27}{4}\right)^{\frac{1}{4}} \approx 1.61$.

To find the minimum of the left-hand side of inequality (9), we consider the function

$$
h(z)=\frac{(z+\check{m})^{z+\check{m}}}{z^{z+1}}, \quad \text { where } z \geq 1 .
$$

For $\check{m}=1$ we have $h(z)=(1+1 / z)^{z+1}$, which is strictly decreasing toward Euler's number $e$ as $z$ increases. For $\check{m} \geq 2$ we show that $h$ is strictly increasing. Indeed, the derivative of $\ln h(z)$ equals

$$
\ln \left(1+\frac{\check{m}}{z}\right)-\frac{1}{z}
$$

This expression is positive for $\check{m} \geq 2$ and $z \geq 1$, since we have

$$
\begin{aligned}
\exp \left(\frac{1}{z}\right) & =1+\frac{1}{z}\left(1+\sum_{k \geq 2} \frac{1}{k ! z^{k-1}}\right) \\
& \leq 1+\frac{1}{z}\left(1+\sum_{k \geq 2} \frac{1}{k !}\right)=1+\frac{e-1}{z}<1+\frac{\check{m}}{z} .
\end{aligned}
$$

For $k=2$ and $m_{2}=1$, the left-hand side of inequality (9) equals $\sqrt{h\left(m_{1}\right)}$ with $\check{m}=1$. It is thus strictly larger than $\sqrt{e} \approx 1.64$, which in turn is larger than $(27 / 4)^{1 / 4}$, the maximum of the right-hand side of inequality (9).

For $k=2$ and $m_{2}>1$, and for $k \geq 3$, the analysis of $h$ implies that the left-hand side of inequality (9) is strictly increasing in each of the variables $m_{i}, 1 \leq i \leq k$, because it equals

$$
\left(\frac{h\left(m_{i}\right)}{\prod_{j \neq i} m_{j}^{m_{j}+1}}\right)^{1 / k}
$$

with $\breve{m}=\sum_{j \neq i} m_{j}$. For $k=2$ and $m_{2}>1$, this expression is minimised at $m_{1}=1$, where it is larger than $\sqrt{e}$, as already shown. For $k>2$ the minimum is attained at $m_{1}=\cdots=m_{k}=1$, and is equal to $k$.

\subsection{The bound for standard Young tableaux of straight shape}

The goal of this subsection is to prove the special case of Theorem 31 where $\mu=\emptyset$ and $k=1$. Note that we have the equivalence

$$
|\operatorname{BST}(\lambda, 1)| \geq \sum_{d \mid n, d>1}|\operatorname{BST}(\lambda, d)| \Longleftrightarrow \frac{\sum_{d \mid n}|\operatorname{BST}(\lambda, d)|}{|\operatorname{BST}(\lambda, 1)|} \leq 2 .
$$

For the remainder of this subsection we focus on proving the latter inequality for $\lambda \notin\left\{(n),\left(1^{n}\right)\right\}$. We shall first make use of the following theorem by Fomin and Lulov: 
Theorem 37 ([FL97]). For any partition $\lambda \vdash n$, we have

$$
|\operatorname{BST}(\lambda, d)| \leq Q(n, d) \cdot|\operatorname{BST}(\lambda, 1)|^{1 / d}, \quad \text { where } \quad Q(n, d):=\sqrt[d]{\frac{d^{n}}{\left(\begin{array}{c}
n \\
n / d, \ldots, n / d
\end{array}\right)}} .
$$

We introduce the auxiliary function $B_{n}(x)$ as

$$
B_{n}(x):=\sum_{d \mid n} Q(n, d) x^{\frac{1}{d}-1}
$$

By plugging $x=|\operatorname{BST}(\lambda, 1)|$ into definition (12), and using formula (11), we have

$$
\begin{aligned}
B_{n}(|\operatorname{BST}(\lambda, 1)|) & =\sum_{d \mid n} Q(n, d)|\operatorname{BST}(\lambda, 1)|^{\frac{1}{d}-1} \\
& =\frac{\sum_{d \mid n} Q(n, d)|\operatorname{BST}(\lambda, 1)|^{1 / d}}{|\operatorname{BST}(\lambda, 1)|} \\
& \geq \frac{\sum_{d \mid n}|\operatorname{BST}(\lambda, d)|}{|\operatorname{BST}(\lambda, 1)|}
\end{aligned}
$$

Hence, if we can show that $B_{n}(x) \leq 2$ for suitable values of $x$ and $n$, we obtain the second inequality in formula (10).

Lemma 38. The inequality

$$
\frac{\sum_{d \mid n}|\operatorname{BST}(\lambda, d)|}{|\operatorname{BST}(\lambda, 1)|} \leq 2
$$

holds for all partitions $\lambda \vdash n$ other than $(n)$ and $\left(1^{n}\right)$ with $n$ composite.

Proof. When $n$ is a prime number, the statement is trivial. Otherwise, we distinguish between several cases.

Case $\lambda=(n-1,1)$ or $\lambda=\left(2,1^{n-2}\right)$. In this case,

$$
|\operatorname{BST}(\lambda, d)|= \begin{cases}n-1 & \text { if } d=1 \\ 1 & \text { otherwise }\end{cases}
$$

and we observe that $n-1 \geq \tau(n)-1$, where $\tau(n)$ is the number of divisors of $n$.

Case $|\lambda| \leq 8$. The remaining 14 partitions (and their conjugates) not covered previously can be verified by hand.

Case $|\lambda| \geq 9$. As noted before, it suffices to show the bound $B_{n}(|\operatorname{BST}(\lambda, 1)|) \leq 2$. To do so we prove the following three statements for $n \geq 9$ :

$\circ$ the function $x \mapsto B_{n}(x)$ is strictly decreasing for fixed $n$,

○ $B_{n}\left(\frac{n^{2}}{3}\right) \leq 2$, and

○ $|\operatorname{BST}(\lambda, 1)| \geq \frac{n^{2}}{3}$ for $\lambda \notin\left\{(n),\left(1^{n}\right),(n-1,1),\left(2,1^{n-2}\right)\right\}$.

The first item is obvious from the definition of $B_{n}(x)$, as all exponents of $x$ are negative. The second item is proved later in Lemma 40 . We proceed by verifying the third item.

Suppose that $|\lambda| \geq 9$ and that $\lambda$ is not one of the excluded shapes. We will prove the statement by induction on the size of $\lambda$. To be able to perform the inductive step, we first consider the following four exceptional shapes. If $\lambda$ is a hook of the form $\left(n-2,1^{2}\right)$ or its conjugate, then $|\operatorname{BST}(\lambda, 1)|=\left(\begin{array}{c}n-1 \\ 2\end{array}\right)$. Moreover, if $\lambda=(n-2,2)$ or its conjugate, then $|\operatorname{BST}(\lambda, 1)|=\frac{n(n-3)}{2}$. In both cases, the inequality is true for $n \geq 9$. 
The base cases of the induction, $|\lambda| \in\{9,10\}$, can be verified using a computer, so we proceed to the inductive step.

If $\lambda$ is a rectangle and $n \geq 11$, we can remove two cells from either the last row or the last column to obtain two new partitions $\mu$ and $v$ of size $n-2$, respectively. A simple bijective argument shows that

$$
|\operatorname{BST}(\lambda, 1)|=|\operatorname{BST}(\mu, 1)|+|\operatorname{BST}(\nu, 1)| \text {. }
$$

By induction, $|\operatorname{BST}(\lambda, 1)| \geq 2(n-2)^{2} / 3 \geq n^{2} / 3$, where the last inequality is true for $n \geq 7$. Note that $\mu$ and $v$ are not among the excluded shapes.

If $\lambda$ is not a rectangle and is not an exceptional shape, we obtain two partitions $\mu$ and $\nu$ of size $n-1$ by removing two distinct corners of $\lambda$. This is possible because $\lambda$ is not a rectangle.

These partitions $\mu$ and $v$ are not among the excluded shapes, because $\lambda$ is not one of the exceptional shapes. Therefore we have

$$
|\operatorname{BST}(\lambda, 1)| \geq|\operatorname{BST}(\mu, 1)|+|\operatorname{BST}(\nu, 1)| .
$$

The inequality follows as before by induction.

Lemma 39. For positive integers $d \mid n$, we have

$$
Q(n, d) \leq \sqrt{n},
$$

where $Q(n, d)=\left(\frac{d^{n}}{n ! /((n / d) !)^{d}}\right)^{1 / d}$ is as in formula $(11)$.

Proof. First we prove formula (13) for $n \in\{1,2\}$ by direct inspection. For $n \geq 3$, we again use Robbins' approximation (Proposition 34). We have

$$
\begin{aligned}
Q(n, d) & =\left(\frac{d^{n}}{n ! /((n / d) !)^{d}}\right)^{\frac{1}{d}}=\frac{d^{n / d}(n / d) !}{(n !)^{1 / d}} \\
& =(2 \pi n)^{-\frac{1}{2 d}}\left(2 \pi \frac{n}{d}\right)^{\frac{1}{2}} \exp \left(r_{n / d}-\frac{r_{n}}{d}\right) .
\end{aligned}
$$

By plugging this into formula (13) and simplifying the expressions, it suffices to show that

$$
\exp \left(2 n d r_{n / d}-2 n r_{n}\right) \leq\left(2 \pi n M_{d}\right)^{n},
$$

where $M_{d}:=\left(\frac{d}{2 \pi}\right)^{d}$. Now using the approximations $r_{n / d}<\frac{d}{12 n}$ and $r_{n}>0$, we have

$$
2 n d r_{n / d}-2 n r_{n} \leq 2 n d r_{n / d} \leq \frac{2 n d^{2}}{12 n}=\frac{d^{2}}{6},
$$

and thus the left-hand side of formula (14) can be bound with exp $\left(\frac{d^{2}}{6}\right)$.

Claim: For positive integers $n \geq 3, n \geq d$, we have

$$
\exp \left(\frac{d^{2}}{6}\right) \leq\left(2 \pi n M_{d}\right)^{n}
$$

Case $d \geq 8$. In this case we have $\exp (1 / 6)<\frac{d}{2 \pi}$, and therefore $\exp \left(\frac{d^{2}}{6}\right)<M_{d}^{d} \leq M_{d}^{n} \leq\left(2 \pi n M_{d}\right)^{d}$. This proves formula (15).

Case $1 \leq d \leq 7$. Direct inspection shows that $2 \pi M_{d} \geq 2 \pi M_{2}=2 / \pi$, so for $n \geq 2$ we have $2 \pi n M_{d}>1$ and therefore $\left(2 \pi n M_{d}\right)^{n}$ is strictly increasing in $n$. 
Finally, direct inspection now verifies that formula (15) is already satisfied for $n=3$ and each $d \in\{1, \ldots, 7\}$. This proves the claim and concludes the proof.

Lemma 40. For integers $n \geq 9$, we have $B_{n}\left(\frac{n^{2}}{3}\right) \leq 2$.

Proof. We first verify the inequality for all $n$ in the range $9 \leq n \leq 120$. This can be done using a computer. For $n \geq 121$, we shall bound $B_{n}(x)$ from above by $g_{n}(x)$, defined as

$$
g_{n}(x)=1+\sqrt{n} x^{-\frac{1}{2}}+2 n x^{-\frac{2}{3}} .
$$

A simple computation shows that for $n=121$, we have $g_{n}\left(\frac{n^{2}}{3}\right) \approx 1.999$. Hence, if we can show that ○ $B_{n}(x) \leq g_{n}(x)$ for all $x \geq 1$ and $\circ n \mapsto g_{n}\left(\frac{n^{2}}{3}\right)$ is strictly decreasing,

then we are done. We proceed with the first point. Recall that the number of divisors of $n$ is at most $2 \sqrt{n}$ for $n \geq 1$. We thus obtain

$$
\begin{aligned}
B_{n}(x) & =\sum_{d \mid n} Q(n, d) x^{\frac{1}{d}-1} \\
& =1+\sum_{d \mid n, d>1} Q(n, d) x^{\frac{1}{d}-1} \\
\{\text { by Lemma } 39\} & \leq 1+\sum_{d \mid n, d>1} \sqrt{n} x^{\frac{1}{d}-1} \\
& \leq 1+\sqrt{n} x^{-\frac{1}{2}}+\sqrt{n} \sum_{d \mid n, d>1} x^{\frac{1}{3}-1} \\
& \leq 1+\sqrt{n} x^{-\frac{1}{2}}+2 n x^{-\frac{2}{3}} \\
& =g_{n}(x) .
\end{aligned}
$$

For the second point, it is enough to note that

$$
g_{n}\left(n^{2} / 3\right)=1+\frac{\sqrt{3}}{\sqrt{n}}+\frac{2 \times 9^{1 / 3}}{\sqrt[3]{n}},
$$

which is obviously decreasing in $n$.

\subsection{The bound for skew standard Young tableaux}

We now extend the result of the previous section to skew shapes.

Lemma 41. Let $\lambda / \mu$ be a skew shape with $n$ cells. Then

$$
|\operatorname{BST}(\lambda / \mu, 1)| \geq \sum_{d \mid n, d>1}|\operatorname{BST}(\lambda / \mu, d)|
$$

if and only if $\lambda / \mu$ is neither the partition $(n)$ nor the partition $\left(1^{n}\right)$.

Proof. We distinguish two cases:

Case 1. $\lambda / \mu$ has at least two cells in some row and in some column. 
We prove the following sequence of inequalities:

$$
\begin{aligned}
|\operatorname{BST}(\lambda / \mu, 1)| & =\sum_{\nu \vdash n} c_{\mu, v}^{\lambda}|\operatorname{BST}(\nu, 1)| \\
& \geq \sum_{\nu \vdash n} c_{\mu, v}^{\lambda} \sum_{d \mid n, d>1}|\operatorname{BST}(v, d)| \\
& \geq \sum_{d \mid n, d>1}|\operatorname{BST}(\lambda / \mu, d)| .
\end{aligned}
$$

We first prove inequality (16), for each summand separately. That is, we show that for all partitions $v \vdash n$,

$$
c_{\mu, v}^{\lambda}|\operatorname{BST}(v, 1)| \geq c_{\mu, v}^{\lambda} \sum_{d \mid n, d>1}|\operatorname{BST}(v, d)| .
$$

Since $\lambda / \mu$ has at least two cells in the same row and two cells in the same column, we can apply Lemma 8. It follows that $c_{\mu,\left(1^{n}\right)}^{\lambda}=c_{\mu,(n)}^{\lambda}=0$. Thus, the inequality holds for $v=\left(1^{n}\right)$ and $(n)$. For all other partitions $v \vdash n$, the inequality follows from Lemma 38 .

We now change the order of summation and prove inequality (17), again separately for each summand. That is, for fixed $d>1$ with $d m=n$, we show

$$
\sum_{\nu \vdash n} c_{\mu, \nu}^{\lambda}|\operatorname{BST}(\nu, d)| \geq|\operatorname{BST}(\lambda / \mu, d)| .
$$

Indeed, we have

$$
\begin{aligned}
\sum_{\nu \vdash n} c_{\mu, v}^{\lambda}|\operatorname{BST}(v, d)| & =\sum_{\nu \vdash n} c_{\mu, v}^{\lambda}\left|\chi^{\nu}\left(\left(d^{m}\right)\right)\right| \\
& \geq\left|\chi^{\lambda / \mu}\left(\left(d^{m}\right)\right)\right| \\
& =|\operatorname{BST}(\lambda / \mu, d)| .
\end{aligned}
$$

The two equalities follow from Corollary 30, whereas the inequality is obtained by taking absolute values on both sides of the expansion of the skew character into irreducible characters (equation (3)), evaluated at $\left(d^{m}\right)$ and applying the triangle inequality.

Case 2. All columns or all rows of $\lambda / \mu$ contain at most one cell.

By symmetry, we may assume that every connected component of $\lambda / \mu$ is a single row. Let the lengths of these rows be $n_{1}, n_{2}, \ldots, n_{r}$. We have $\operatorname{BST}(\lambda / \mu, d)=\emptyset$ unless all $n_{i}$ are multiples of $d$. In this case we find by explicit enumeration that

$$
|\operatorname{BST}(\lambda / \mu, d)|=\left(\begin{array}{c}
n / d \\
n_{1} / d, n_{2} / d, \ldots, n_{r} / d
\end{array}\right) .
$$

It then suffices to prove that

$$
\left(\begin{array}{c}
n \\
n_{1}, n_{2}, \ldots, n_{r}
\end{array}\right) \geq \sum_{d>1, d \mid \operatorname{gcd}\left(n_{1}, n_{2}, \ldots, n_{r}\right)}\left(\begin{array}{c}
n / d \\
n_{1} / d, n_{2} / d, \ldots, n_{r} / d
\end{array}\right) .
$$

This inequality is an easy consequence of Corollary 36.

\subsection{The general case}

We are now ready to prove Theorem 31 itself, which we restate for convenience. 
Theorem. Let $\lambda / \mu$ be a skew shape with $n$ cells and let $k$ be a positive integer with $k \mid n$. Suppose that $|\operatorname{BST}(\lambda / \mu, k)| \geq 2$. Then

$$
|\operatorname{BST}(\lambda / \mu, k)| \geq \sum_{d \mid \frac{n}{k}, d>1}|\operatorname{BST}(\lambda / \mu, d k)| .
$$

Additionally, the inequality holds if $n / k$ is a prime number.

Proof. Let $\left(v^{1} / \kappa^{1}, \ldots, v^{k} / \kappa^{k}\right)$ be the skew $k$-quotient of $\lambda / \mu$. We first establish, for $1 \leq i \leq k$, the inequality

$$
\left(\tau\left(\left|v^{i} / \kappa^{i}\right|\right)-1\right)\left|\operatorname{BST}\left(v^{i} / \kappa^{i}, 1\right)\right| \geq \sum_{\substack{d \| v^{i} / \kappa^{i} \mid \\ d>1}}\left|\operatorname{BST}\left(v^{i} / \kappa^{i}, d\right)\right|
$$

If $v^{i} / \kappa^{i}$ is neither the single-row nor the single-column partition, the bound for skew standard Young tableaux (Lemma 41) applies. Moreover, in this case $\left|v^{i} / \kappa^{i}\right| \geq 3$, and therefore $\tau\left(\left|v^{i} / \kappa^{i}\right|\right)-1 \geq 1$. Otherwise, $\left|\operatorname{BST}\left(v^{i} / \kappa^{i}, d\right)\right|=1$ for all $d|| v^{i} / \kappa^{i} \mid$, and the inequality holds trivially.

Thus, setting $g=\operatorname{gcd}\left(\left|v^{1} / \kappa^{1}\right|, \ldots,\left|v^{k} / \kappa^{k}\right|\right)$,

$$
\begin{aligned}
& \prod_{\substack{i=1 \\
v^{i} / \kappa^{i} \neq \emptyset}}^{k}\left(\tau\left(\left|v^{i} / \kappa^{i}\right|\right)-1\right)\left|\operatorname{BST}\left(v^{i} / \kappa^{i}, 1\right)\right| \geq \prod_{\substack{i=1 \\
v^{i} / \kappa^{i} \neq \emptyset}}^{k} \sum_{\substack{d \| v^{i} / \kappa^{i} \mid \\
d>1}}\left|\operatorname{BST}\left(v^{i} / \kappa^{i}, d\right)\right| \\
& \geq \sum_{\substack{d \mid g \\
d>1}} \prod_{\substack{i=1 \\
v^{i} / \kappa^{i} \neq \emptyset}}^{k}\left|\operatorname{BST}\left(v^{i} / \kappa^{i}, d\right)\right| \\
& =\sum_{\substack{d \mid g \\
d>1}} \prod_{i=1}^{k}\left|\operatorname{BST}\left(v^{i} / \kappa^{i}, d\right)\right| \\
& \left\{\text { by Theorem 22\} }=\sum_{\substack{d \mid g \\
d>1}} \frac{|\mathrm{BST}(\lambda / \mu, d k)|}{\left(\begin{array}{c}
\sum_{i}\left|v^{i} / \kappa^{i}\right| / d, \ldots,\left|v^{k} / \kappa^{k}\right| / d \\
(1)
\end{array}\right.}\right. \text {. }
\end{aligned}
$$

Note that for any $d \geq 1$, there is exactly one border-strip tableau having empty shape: $|\operatorname{BST}(\emptyset, k)|=1$. Suppose that $g>1$ and there are at least two nonempty skew shapes among $v^{1} / \kappa^{1}, \ldots, v^{k} / \kappa^{k}$. Then we can apply this inequality and Corollary 36 and obtain

$$
\begin{aligned}
|\operatorname{BST}(\lambda / \mu, k)| & =\left(\begin{array}{c}
\sum_{i}\left|v^{i} / \kappa^{i}\right| \\
\left|v^{1} / \kappa^{1}\right|, \ldots,\left|v^{k} / \kappa^{k}\right|
\end{array}\right) \prod_{i=1}^{k}\left|\operatorname{BST}\left(v^{i} / \kappa^{i}, 1\right)\right| \\
& \geq\left(\begin{array}{c}
\sum_{i}\left|v^{i} / \kappa^{i}\right| \\
\left|v^{1} / \kappa^{1}\right|, \ldots,\left|v^{k} / \kappa^{k}\right|
\end{array}\right) \prod_{\substack{i=1 \\
v^{i} / \kappa^{i} \neq \emptyset}}^{k}\left(\tau\left(\left|v^{i} / \kappa^{i}\right|\right)-1\right)^{-1} \\
& \sum_{d \mid g, d>1}|\operatorname{BST}(\lambda / \mu, d k)|\left(\begin{array}{c}
\sum_{i}\left|v^{i} / \kappa^{i}\right| / d \\
\left|v^{1} / \kappa^{1}\right| / d, \ldots,\left|v^{k} / \kappa^{k}\right| / d
\end{array}\right)^{-1} \\
& =\sum_{d \mid g, d>1}|\operatorname{BST}(\lambda / \mu, d k)| \\
& =\operatorname{BST}(\lambda / \mu, d k) \mid
\end{aligned}
$$


If $g=1$, the inequality is trivially true. If there is precisely one nonempty skew shape $v / \kappa$ among $v^{1} / \kappa^{1}, \ldots, v^{k} / \kappa^{k}$, we have $|\operatorname{BST}(\lambda / \mu, d k)|=|\operatorname{BST}(v / \kappa, d)|$ for all $d$ by Theorem 22 . If $v / \kappa$ is neither $(n / k)$ nor $\left(1^{n / k}\right)$, Lemma 41 applies and we have

$$
|\operatorname{BST}(\lambda / \mu, k)|=|\operatorname{BST}(\nu / \kappa, 1)| \geq \sum_{d \mid \frac{n}{k}, d>1}|\operatorname{BST}(v / \kappa, d)|=\sum_{d \mid \frac{n}{k}, d>1}|\operatorname{BST}(\lambda / \mu, d k)| .
$$

Otherwise, if $v / \kappa$ is either $(n / k)$ or $\left(1^{n / k}\right)$, Corollary 25 implies that there is only one element in $\operatorname{BST}(\lambda / \mu, k)$.

\section{Cyclic sieving for skew standard tableaux}

In this section we apply the bounds established in the previous section and Theorem 3 to prove the existence of several cyclic sieving phenomena for various families of skew standard Young tableaux.

Let us first put the bound from Theorem 31 into the form required to apply Theorem 3.

Proposition 42. Let $\lambda / \mu$ be a nonempty skew shape with $n$ cells, set $m \in \mathbb{N}_{0}$ and let $k$ be a positive integer with $k \mid n$. Then

$$
\sum_{d \mid \frac{n}{k}} \mu(d)|\operatorname{BST}(\lambda / \mu, d k)|^{m} \geq 0
$$

or equivalently,

$$
\sum_{d \mid k} \mu(k / d)\left|f^{\lambda / \mu}\left(\xi^{d}\right)\right|^{m} \geq 0
$$

for a primitive nth root of unity $\xi$.

Proof. The equivalence follows from Corollary 30 and replacing $d$ with $\frac{n}{d k}$ and $k$ with $\frac{n}{k}$. We prove the first inequality.

If $|\operatorname{BST}(\lambda / \mu, k)|=1$, we also have $|\operatorname{BST}(\lambda / \mu, d k)|=1$ for any $d \mid \frac{n}{k}$ by Corollary 25. Therefore,

$$
\sum_{d \mid \frac{n}{k}} \mu(d)|\operatorname{BST}(\lambda / \mu, d k)|^{m}=\sum_{d \mid \frac{n}{k}} \mu(d)=\left\{\begin{array}{ll}
1 & \text { if } n=k \\
0 & \text { if } n \neq k
\end{array} \geq 0 .\right.
$$

This reasoning also covers the case $m=0$.

Otherwise, since $\mu(1)=1$ and $\mu(d) \geq-1$, we have

$$
\sum_{d \mid \frac{n}{k}} \mu(d)|\operatorname{BST}(\lambda / \mu, d k)|^{m} \geq|\operatorname{BST}(\lambda / \mu, k)|^{m}-\sum_{d \mid \frac{n}{k}, d>1}|\operatorname{BST}(\lambda / \mu, d k)|^{m} \geq 0,
$$

where the final inequality follows from Theorem 31 .

Remark 43. One might think that $\left|f^{\lambda / \mu}\left(\xi^{d}\right)\right|$ could be the number of fixed points of a group action, despite the fact that $\left|f^{\lambda / \mu}(q)\right|$ is not a polynomial. However, this is not the case.

For example, consider $\lambda=(2,1)$. Then $f^{\lambda}(q)=q+q^{2}$ and, for a 3rd root of unity $\xi$, we have $\left|f^{\lambda}\left(\xi^{3}\right)\right|=|\operatorname{BST}(\lambda, 1)|=2$ and $\left|f^{\lambda}(\xi)\right|=|\operatorname{BST}(\lambda, 3)|=1$. This is incompatible with the possible orbit sizes of a group action on a set with two elements. Indeed, for $k=3$ we obtain

$$
\frac{1}{k} \sum_{d \mid k} \mu(k / d)\left|f^{\lambda}\left(\xi^{d}\right)\right|=\frac{1}{3}(-1+2),
$$

which, by Remark 5, would have to be an integer.

Taking into account the previous remark, it makes sense to look for shapes $\lambda / \mu$ such that the character $f^{\lambda / \mu}$ evaluated at roots of unity is nonnegative. 
Theorem 44. Let $\lambda / \mu$ be a skew shape with $n$ cells and let $m$ be a positive integer. Then there is a cyclic group action $\rho$ of order $n$ such that

$$
(\underbrace{\operatorname{SYT}(\lambda / \mu) \times \cdots \times \operatorname{SYT}(\lambda / \mu)}_{m},\langle\rho\rangle, f^{\lambda / \mu}(q)^{m})
$$

exhibits the cyclic sieving phenomenon if and only if $m$ is even, or $m$ is odd and for each positive integer $k$ with $k \mid n$ every tiling of $\lambda / \mu$ with strips of size $k$ has even height.

Remark 45. The case $m=2$ of this theorem does not extend to squares of arbitrary representations of the symmetric group. For example, consider the representation with character $\chi^{(4)}+\chi^{\left(2,1^{2}\right)}$. Its fake-degree polynomial is $f(q)=1+q^{3}+q^{4}+q^{5}$. Then we obtain, for a primitive fourth root of unity $\xi$, that $f(\xi)^{2}=4$ and $f\left(\xi^{2}\right)^{2}=0$. This violates the condition in Theorem 3 for $k=2$, because $\mu(2) f(\xi)^{2}+\mu(1) f\left(\xi^{2}\right)^{2}=-4$.

Proof. Let $\xi$ be an $n$th primitive root of unity. Then Proposition 42, together with Theorem 3, ensures the existence of $\rho$, provided that $f^{\lambda / \mu}\left(\xi^{d}\right)^{m}$ is nonnegative for all $d \mid n$. Conversely, nonnegativity is a necessary condition because, given a cyclic group action $\rho$, the number of fixed points of $\rho^{d}$ equals $f^{\lambda / \mu}\left(\xi^{d}\right)^{m}$.

It remains to consider the case of odd $m$. By Corollary $30, f^{\lambda / \mu}\left(\xi^{d}\right)$, with $d=\frac{n}{k}$, is nonnegative if and only if every tiling of $\lambda / \mu$ with strips of size $k$ has even height.

Corollary 46. Let $\lambda=\left(a, 1^{n-a}\right)$ be a hook-shaped partition of $n$. Then there is a cyclic group action $\rho$ such that $\left(\operatorname{SYT}(\lambda),\langle\rho\rangle, f^{\lambda}(q)\right)$ exhibits the cyclic sieving phenomenon if and only if $n$ and a are odd and $a-1 \bmod m$ is even for $m \mid n, 1 \leq m<a$.

Proof. Suppose that $n$ and $a$ are odd, and $m \mid n$. In particular, $m$ is odd too. Note that there is a unique tiling of a hook with border strips of size $m$. We have to show that the height of this tiling is even if and only if $a-1 \bmod m$ is even.

Recall that the height of a tile is one less than the number of rows it spans. If and only if $a-1 \bmod m$ is even, the height of the tile covering the top left corner of the shape must be even: this tile must cover an odd number of cells in the first row and, since its size $m$ is odd, an even number of cells in the first column. Since the height of all other tiles is evidently even too, so is the total height.

If the parity of $n$ and $a$ is different, then the tiling with a single strip of size $n$ has height $n-a$, which is odd. If both $n$ and $a$ are even, the tiling with two strips of size $n / 2$ has odd height: if $a \leq n / 2$, the height is $n-a-1$, otherwise the height is $n-1$.

Remark 47. According to the previous result, for $\lambda=\left(3,1^{n-3}\right)$, a cyclic group action of order $n$ with character $f^{\lambda}(q)=q^{(n-2)(n-3) / 2} \frac{[n-1]_{q}[n-2]_{q}}{[2]_{q}}$ exists for all odd $n>3$. In this case, there should be one singleton orbit and $(n-3) / 2$ orbits of size $n$. Indeed, an appropriate group action can be constructed as follows:

Identify a tableau with the two labels $x<y$ different from 1 in the first row. Note that $y-x \in$ $\{1,2, \ldots, n-2\}$, and only the pair $(2, n)$ has difference $n-2$. We let the generator of the group action $\eta$ act as follows:

$$
\eta(x, y):= \begin{cases}(2, n) & \text { if } x=2, y=n, \\ (x+2, y+2) & \text { if } \leq x<y \leq n-2, \\ (2, x+1) & \text { if } y=n-1 \\ (3, x+1) & \text { if } x>2, y=n .\end{cases}
$$

We then note that if $(u, v)=\eta(x, y)$, then $v-u \in\{y-x,(n-2)-(y-x)\}$. This explains why there are $(n-3) / 2$ orbits of length $n$. We leave the remaining details to the reader. 
Remark 48. It turns out that one can determine the number of border strips $\lambda / \mu$ of size $n$ which carry a cyclic group action of order $n$ and character $f^{\lambda / \mu}(q)$. This will appear in a separate note [Pfa20].

A different way to ensure positivity of the character $f^{\lambda / \mu}$ is to decrease the order of the cyclic group, as in Remark 2.

Theorem 49. Let $\lambda / \mu$ be a skew shape such that every row contains a multiple of $m$ cells. Then there is a cyclic group action $\rho$ of order $m$ such that

$$
\left(\operatorname{SYT}(\lambda / \mu),\langle\rho\rangle, f^{\lambda / \mu}(q)\right)
$$

exhibits the cyclic sieving phenomenon.

Proof. By Theorem 3, it suffices to show that for a primitive $m$ th root of unity $\zeta$ and every $k \mid m$,

$$
\sum_{d \mid k} \mu(k / d) f^{\lambda / \mu}\left(\zeta^{d}\right) \geq 0
$$

Let $|\lambda / \mu|=d m$. By Proposition 42, we have

$$
\sum_{d \mid k} \mu(k / d)\left|f^{\lambda / \mu}\left(\xi^{d}\right)\right| \geq 0
$$

for an $n$th root of unity $\xi$ and every $k \mid d m$. Let $\zeta=\xi^{\frac{n}{m}}$. Then by Corollary 30,

$$
f^{\lambda / \mu}\left(\zeta^{d}\right)=f^{\lambda / \mu}\left(\xi^{d \frac{n}{m}}\right)=(-1)^{\text {height }(B)}\left|\operatorname{BST}\left(\lambda / \mu, \frac{m}{d}\right)\right|
$$

Since the length of every row of $\lambda / \mu$ is a multiple of $m$, there is a filling with border strips of size $\frac{m}{d} \mid m$, where every strip has height 0 .

We remark that stretching shapes seems to be a fruitful way to construct cyclic sieving phenomena, as was previously shown with fillings related to Macdonald polynomials by Alexandersson and Uhlin [AU20]. We also mention the following conjecture, which has recently been proved in the nonskew case in [OP20, Cor. 3.3]:

Conjecture 50 ([AA19, Conj. 3.4]). There is an action $\beta$ on the set of semistandard Young tableaux $\operatorname{SSYT}(m \lambda / m \mu, k)$ of order $m$ such that

$$
\left(\operatorname{SSYT}(m \lambda / m \mu, k),\langle\beta\rangle, \mathrm{s}_{m \lambda / m \mu}\left(1, q, q^{2}, \ldots, q^{k-1}\right)\right)
$$

exhibits the cyclic sieving phenomenon.

For some shapes $\lambda / \mu$, the tiling may have odd height, but one can multiply $f^{\lambda / \mu}(q)$ with $q^{n / 2}$, provided that the size $n$ of $\lambda / \mu$ is even, to obtain positivity at roots of unity. An important example is the case of rectangular shapes. In this case, Rhoades has proved that promotion together with a natural $q$-analogue of the hook length formula exhibits the cyclic sieving phenomenon. The following result is much weaker, because it only establishes the existence of a group action, but it is also much easier to prove, and illustrates the method. 
Theorem 51 ([Rho10a]). Let $\lambda=a^{b}$ be a rectangular diagram with $n=$ ab cells, and set $\kappa(\lambda):=\sum_{j}\left(\begin{array}{c}\lambda_{j}^{\prime} \\ 2\end{array}\right)$. Then there is a cyclic group action $\partial$ of order $n$ such that

$$
\left(\operatorname{SYT}(\lambda),\langle\partial\rangle, q^{-\kappa(\lambda)} f^{\lambda}(q)\right)
$$

exhibits the cyclic sieving phenomenon.

Proof. It is a well-known result by Stanley [Sta01, Cor. 7.21.5] that

$$
q^{-\kappa(\lambda)} f^{\lambda}(q)=\frac{[n]_{q} !}{\prod_{\square \in \lambda}[h(\square)]_{q}}
$$

where $h(\square)$ is the hook value of $\square$. In particular, $q^{-\kappa(\lambda)} f^{\lambda}(q)$ is a polynomial. We must check that this is nonnegative whenever $q$ is an $n$th root of unity. Suppose that $m \mid n, n=d m$, and let $\xi$ be a primitive $n$th root of unity. Corollary 30 implies that $f^{\lambda}\left(\xi^{d}\right)$ is nonzero if and only if BST $\left(a^{b}, m\right)$ is nonempty. Using the abacus, one can show that $m \mid a$ or $m \mid b$ if and only if the $m$-core is empty, which for straight shapes is equivalent to $\left|\operatorname{BST}\left(a^{b}, m\right)\right|>0$. From here, it is a straightforward exercise to show that $\kappa(\lambda)=b a(a-1) / 2$ and that $\xi^{-d \cdot b a(a-1) / 2} f^{\lambda}\left(\xi^{d}\right)$ is nonnegative for all $d \mid n$.

Finally, Proposition 42 and Theorem 3 give the result.

\section{Permutations and invariants of the adjoint representation of $\mathbf{G L}_{\boldsymbol{n}}$}

In this section we apply our results to study the space of invariants of tensor powers of the adjoint representation $\mathfrak{g l}_{r}$ of the general linear group $\mathrm{GL}_{r}$.

Definition 52. The rotation $\operatorname{rot} \sigma$ of a permutation $\sigma \in \mathbb{S}_{n}$ is the permutation obtained by conjugating with the long cycle $(1, \ldots, n)$.

Remark 53. Equivalently, if $M_{\sigma}$ is the permutation matrix corresponding to $\sigma$, then $M_{\text {rot } \sigma}$ is obtained by removing the first column of $M_{\sigma}$ and appending it on the right, then removing the first row and appending it at the bottom.

Still equivalently, let $D_{\sigma}$ be the chord diagram associated with $\sigma$ - that is, the directed graph with vertices $\{1, \ldots, n\}$ arranged counterclockwise on a circle, and $\operatorname{arcs}(i, \sigma(i))$. Then $D_{\text {rot } \sigma}$ is the chord diagram obtained by rotating the graph clockwise. See Figure 5 for an illustration.

The following theorem makes the character of rotation explicit:

Theorem 54 ([BRS08, Rho10b, RW14]).

$$
\left(\Im_{n},\langle\operatorname{rot}\rangle, \sum_{\lambda \vdash n} f^{\lambda}(q)^{2}\right)
$$

exhibits the cyclic sieving phenomenon.

Proof. Consider the adjoint representation of $\mathfrak{S}_{n}-$ that is, $\mathfrak{S}_{n}$ acting on itself by conjugation, or relabelling. It is well known (see, for example, [Sta01, Ex. 7.71a]) that the character of this representation equals $\sum_{\lambda \vdash n} \chi^{\lambda} \bar{\chi}^{\lambda}$. Since the restriction of the adjoint representation to the cyclic group generated by the long cycle $(1, \ldots, n)$ is precisely the action rot, the result follows from Proposition 11 .

Definition 55. Recall that the Robinson-Schensted correspondence provides a bijection

$$
\mathfrak{S}_{n} \leftrightarrow\{(P, Q) \in \operatorname{SYT}(\lambda) \times \operatorname{SYT}(\lambda): \lambda \vdash n\}
$$

The shape $\operatorname{sh}(\sigma)$ of a permutation $\sigma$ is the common shape of the standard Young tableaux $P$ and $Q$ corresponding to $\sigma$ under the Robinson-Schensted correspondence. We let $R_{\lambda}$ denote the set of permutations of shape $\lambda$. 
$[5,4,1,2,3]$

$\left(\begin{array}{c|cccc}\hline 0 & 0 & 1 & 0 & 0 \\ \hline 0 & 0 & 0 & 1 & 0 \\ 0 & 0 & 0 & 0 & 1 \\ 0 & 1 & 0 & 0 & 0 \\ 1 & 0 & 0 & 0 & 0 \\ \hline & & & \end{array}\right)$

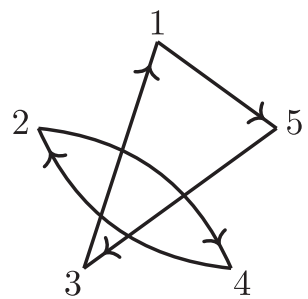

$[3,5,1,2,4]$

$\left(\begin{array}{llll|l}\hline 0 & 0 & 1 & 0 & 0 \\ 0 & 0 & 0 & 1 & 0 \\ 1 & 0 & 0 & 0 & 0 \\ 0 & 0 & 0 & 0 & 1 \\ \hline \hline 0 & 1 & 0 & 0 & 0\end{array}\right)$

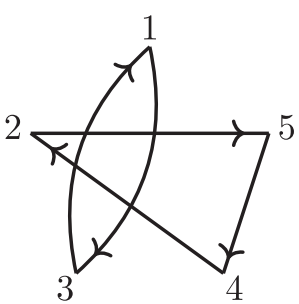

Figure 5. Rotation of $\pi=[5,4,1,2,3]$ as conjugation by the long cycle $(1,2,3,4,5)$, cyclic shift of the permutation matrix and rotation of the chord diagram. Note that $\operatorname{sh}([5,4,1,2,3])=\left(3,1^{2}\right)$ and $\operatorname{sh}([3,5,1,2,4])=(3,2)$.

We are now ready to prove the first major result of this section.

Corollary 56. Let $P_{n}$ be the set of partitions of $n$. Then there exists a map st $: \mathfrak{S}_{n} \rightarrow P_{n}$ which is invariant under rotation and equidistributed with the Robinson-Schensted shape. That is,

$$
\text { st } \circ \text { rot }=\text { st } \quad \text { and } \quad \sum_{\sigma \in \mathbb{S}_{n}} \mathrm{~s}_{\mathrm{st}(\sigma)}(\mathbf{x})=\sum_{\sigma \in \mathbb{S}_{n}} \mathrm{~s}_{\mathrm{sh}(\sigma)}(\mathbf{x})
$$

Moreover, with $\mathfrak{S}_{n}^{\lambda}:=\left\{\pi \in \mathfrak{S}_{n}: \operatorname{st}(\sigma)=\lambda\right\}$, the triple

$$
\left(\mathfrak{S}_{n}^{\lambda},\langle\operatorname{rot}\rangle, f^{\lambda}(q)^{2}\right)
$$

exhibits the cyclic sieving phenomenon.

We stress that we are unable to present such a statistic explicitly.

Remark 57. Let us remark that the distribution of the shape of a permutation is well studied. Writing $f^{\lambda}$ for the number of standard Young tableaux of shape $\lambda$, we have $\sum_{\sigma \in \mathbb{S}_{n}} \mathrm{~s}_{\operatorname{sh}(\sigma)}(\mathbf{x})=\sum_{\lambda \vdash n}\left(f^{\lambda}\right)^{2} \mathrm{~s}_{\lambda}(\mathbf{x})$. This is closely related to the Plancherel measure, which assigns to each partition $\lambda$ of size $n$ the probability $\left(f^{\lambda}\right)^{2} / n$ ! that a permutation of size $n$, chosen uniformly at random, has shape $\lambda$.

Proof. By Theorem 44, there exists an action of the cyclic group of order $n$ on $R_{\lambda}$ with character $\left(f^{\lambda}(q)\right)^{2}$. Let $\rho$ be the direct sum over all $\lambda \in P_{n}$ of these group actions. Thus, $\left(\mathfrak{S}_{n},\langle\rho\rangle, \sum_{\lambda \vdash n} f^{\lambda}(q)^{2}\right)$ exhibits the cyclic sieving phenomenon. Since $\rho$ acts on each $R_{\lambda}$ separately, we have

$$
\operatorname{sh}(\rho \cdot \sigma)=\operatorname{sh}(\sigma)
$$

By Theorems 7 and 54, the action of $\rho$ and rotation are isomorphic. Therefore we have a bijection $\phi: \mathfrak{S}_{n} \rightarrow \mathfrak{S}_{n}$ with

$$
\phi(\operatorname{rot} \sigma)=\rho \cdot \phi(\sigma)
$$


Defining $\operatorname{st}(\sigma):=\operatorname{sh}(\phi(\sigma))$, we obtain

$$
\operatorname{st}(\operatorname{rot} \sigma)=\operatorname{sh}(\phi(\operatorname{rot} \sigma)) \stackrel{(19)}{=} \operatorname{sh}(\rho \cdot \phi(\sigma)) \stackrel{(18)}{=} \operatorname{sh}(\phi(\sigma))=\operatorname{st}(\sigma) .
$$

Finally we have

$$
\mathfrak{S}_{n}^{\lambda}=\phi^{-1}\left(R_{\lambda}\right),
$$

yielding the last statement.

Remark 58. It is natural to ask whether for $\lambda \vdash n$ we have

$$
\#\left\{\sigma \in R_{\lambda}: \operatorname{rot}^{d}(\sigma)=\sigma\right\}=f^{\lambda}\left(\xi^{d}\right)^{2},
$$

for a positive integer $d$ and a primitive $n$th root of unity $\xi$. In this case, the subset cyclic sieving technique of Alexandersson, Linusson and Potka [ALP19, Prop. 29] would imply the nonskew, $m=2$ case of Theorem 44. However, this fails already for $\lambda=(2,1)$; we have that $R_{\lambda}=\{132,213,231,312\}$ and rot fixes 231 and 312, but $f^{\lambda}(q)^{2}=q^{2}(1+q)^{2}$ evaluates to 1 at $q=\exp (2 \pi i / 3)$.

We now turn to the connection with the invariants of tensor powers of the adjoint representation of $\mathrm{GL}_{r}$, which is the original motivation for this article.

Let $V$ be an $r$-dimensional complex vector space and let $\mathfrak{g l}_{r}=\operatorname{End}(V)$ be the adjoint representation $\mathrm{GL}_{r} \rightarrow \operatorname{End}\left(\mathfrak{g l}_{r}\right), A \mapsto T A T^{-1}$.

The space of $\mathrm{GL}_{r}$-invariants of the $n$th tensor power of $\mathfrak{g l}_{r}$ is $\left(\mathfrak{g l}_{r}^{\otimes n}\right)^{\mathrm{GL}_{r}}=\operatorname{Hom}_{\mathrm{GL}_{r}}\left(\mathfrak{g l}_{r}^{\otimes n}, \mathbb{C}\right)$. A basis for this space can be indexed by Stembridge's alternating tableaux:

Definition 59 ([Ste87]). A staircase is a dominant weight of $\mathrm{GL}_{r}-$ that is, a vector in $\mathbb{Z}^{r}$ with weakly

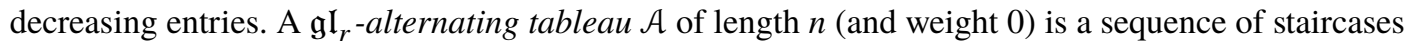

$$
\mathcal{A}=\left(\emptyset=\mu^{0}, \mu^{1}, \ldots, \mu^{2 n}=\emptyset\right)
$$

such that

○ for even $i, \mu^{i+1}$ is obtained from $\mu^{i}$ by adding 1 to an entry, and

$\circ$ for odd $i, \mu^{i+1}$ is obtained from $\mu^{i}$ by subtracting 1 from an entry.

The set of $\mathfrak{g l}_{r}$-alternating tableaux of length $n$ is denoted by $\mathcal{A}_{n}^{(r)}$.

Westbury defined a natural action, promotion, of the cyclic group of order $n$ on the set of socalled invariant words of any finite crystal, in particular alternating tableaux of length $n$, generalising Schützenberger's promotion on rectangular standard Young tableaux. We refrain from giving a definition here and refer to [PRW20] instead.

For our purposes, it is enough to relate promotion to an action on the $\mathrm{GL}_{r}$-invariants of the $n$th tensor power of $\mathfrak{g l}_{r}$. To do so, note that the symmetric group $\mathfrak{S}_{n}$ acts on $\mathfrak{g l} \mathfrak{l}_{r}^{\otimes n}$ by permuting tensor positions, and therefore also on the space of invariants. It turns out that the action of the long cycle $(1, \ldots, n) \in \mathfrak{S}_{n}$ plays a special role:

Theorem 60 ([Wes16, Sec. 6.3]). There is a basis of $\left(\mathfrak{g l}_{r}^{\otimes n}\right)^{\mathrm{GL}}$ which is preserved by the action of the long cycle. Moreover, this action is isomorphic to the action of promotion on the set of alternating tableaux.

Note that Westbury's theorem only asserts the existence of the basis; no explicit construction is known. The main result of this section is the following refinement of his assertion: 
Theorem 61. Let $\mathfrak{S}_{n}^{(r)}:=\left\{\pi \in \mathfrak{S}_{n}: \ell(\operatorname{st}(\pi)) \leq r\right\}=\bigcup_{\substack{\lambda \vdash n \\ \ell(\lambda) \leq r}} \mathfrak{\Xi}_{n}^{\lambda}$. Then there exists a bijection

$$
\mathcal{P}^{(r)}: \mathcal{A}_{n}^{(r)} \rightarrow \mathfrak{S}_{n}^{(r)} \quad \text { with } \quad \mathcal{P}^{(r)} \circ \mathrm{pr}=\operatorname{rot} \circ \mathcal{P}^{(r)}
$$

for $1 \leq r \leq n$.

Equivalently, the action of promotion on the set of $\mathrm{gl}_{r}$-alternating tableaux of length $n$ and the action of rotation on the set of permutations $\mathfrak{\Im}_{n}^{(r)}$ are isomorphic:

$$
\left(\operatorname{pr}, \mathcal{A}_{n}^{(r)}\right) \cong\left(\operatorname{rot}, \mathfrak{S}_{n}^{(r)}\right)
$$

Remark 62. Let us remark that for large dimension - that is, $r \geq n$ - such a bijection was provided by Pfannerer, Rubey and Westbury [PRW20].

Moreover, using the natural injection $i: \mathfrak{S}_{n}^{(r)} \rightarrow \mathfrak{S}_{n}^{(r+1)}$ and the bijections $\mathcal{P}^{(r)}$ and $\mathcal{P}^{(r+1)}$, we define $\iota:=\mathcal{P}^{(r+1)^{-1}} \circ i \circ \mathcal{P}^{(r)}$ and obtain the following commutative diagram:

$$
\begin{array}{cc}
\left(\mathrm{pr}, \mathcal{A}_{n}^{(r)}\right) & \stackrel{\mathcal{P}^{(r)}}{\longrightarrow}\left(\operatorname{rot}, \mathbb{S}_{n}^{(r)}\right) \\
\downarrow^{\iota} & \downarrow^{i} \\
\left(\operatorname{pr}, \mathcal{A}_{n}^{(r+1)}\right) & \stackrel{\mathcal{P}^{(r+1)}}{\longrightarrow}\left(\operatorname{rot}, \mathbb{S}_{n}^{(r+1)}\right) .
\end{array}
$$

In particular, this is an injection $\iota: \mathcal{A}_{n}^{(r)} \rightarrow \mathcal{A}_{n}^{(r+1)}$ such that $\operatorname{pr} \iota(\mathcal{A})=\iota(\operatorname{pr} \mathcal{A})$, which answers the question in [PRW20, rmk. 3.9].

To prove Theorem 61, we first compute a decomposition of the space of invariants as a direct sum of tensor squares of Specht modules.

Lemma 63 ([RW14]). Let $\mathfrak{g l}_{r}$ be the adjoint representation of $\mathrm{GL}_{r}$ and, given a partition $\lambda \vdash n$, let $S_{\lambda}$ be the corresponding irreducible representation of the symmetric group. Then there is an isomorphism of $\mathfrak{S}_{n}$-representations

$$
\left(\mathfrak{g} \mathfrak{l}_{r}^{\otimes n}\right)^{\mathrm{GL}_{r}} \cong \bigoplus_{\substack{\lambda \vdash n \\ \ell(\lambda) \leq r}} S_{\lambda} \otimes S_{\lambda}
$$

Proof. Let $V$ be the vector representation of $\mathrm{GL}_{r}$. Schur-Weyl duality asserts that there is an isomorphism of $\left(\mathrm{GL}_{r} \times \mathfrak{S}_{n}\right)$-representations

$$
V^{\otimes r} \cong \bigoplus_{\substack{\lambda \vdash n \\ \ell(\lambda) \leq r}} V_{\lambda} \otimes S_{\lambda}
$$

where $V_{\lambda}$ is an irreducible representation of $\mathrm{GL}_{r}$.

Recall that by Schur's lemma, $\operatorname{Hom}_{\mathrm{GL}_{r}}\left(V_{\lambda}, V_{\mu}\right)$ contains only the zero map if $\lambda \neq \mu$, and all scalar multiples of the identity otherwise. Thus

$$
\begin{aligned}
\left(\mathfrak{g l}_{r}^{\otimes n}\right)^{\mathrm{GL}_{r}} & \cong \operatorname{Hom}_{\mathrm{GL}_{r}}\left(\left(V \otimes V^{*}\right)^{\otimes n}, \mathbb{C}\right) \\
& \cong \operatorname{End}_{\mathrm{GL}_{r}}\left(V^{\otimes n}\right)
\end{aligned}
$$




$$
\begin{aligned}
\cong \operatorname{End}_{\mathrm{GL}_{r}}\left(\bigoplus_{\substack{\lambda \vdash n \\
\ell(\lambda) \leq r}} V_{\lambda} \otimes S_{\lambda}\right) \\
\{\text { by Schur's lemma }\} \cong \bigoplus_{\substack{\lambda \vdash n \\
\ell(\lambda) \leq r}} \operatorname{End}\left(S_{\lambda}\right) \\
\cong \bigoplus_{\substack{\lambda \vdash n \\
\ell(\lambda) \leq r}} S_{\lambda} \otimes S_{\lambda} .
\end{aligned}
$$

Proof of Theorem 61. By Proposition 11 and Corollary 56, we obtain that the character of $\mathfrak{\subseteq}_{n}^{\lambda}$ equals the character of $\bigoplus_{\substack{\lambda \vdash n \\ \ell(\lambda) \leq r}} S_{\lambda} \otimes S_{\lambda} \downarrow_{\langle(1, \ldots, n)\rangle}$. Summing over all partitions $\lambda$ of length at most $r$, we obtain the character of

$$
\left(\mathfrak{g} \mathfrak{l}_{r}^{\otimes n}\right)^{\mathrm{GL}_{r}} \downarrow_{\langle(1, \ldots, n)\rangle} \cong \bigoplus_{\substack{\lambda \vdash n \\ \ell(\lambda) \leq r}} S_{\lambda} \otimes S_{\lambda} \downarrow_{\langle(1, \ldots, n)\rangle}
$$

by Lemma 63. Therefore, by Brauer's permutation lemma (Theorem 7) and Westbury's theorem (Theorem 60), the cyclic group actions

$$
\left(\operatorname{pr}, \mathcal{A}_{n}^{(r)}\right) \cong\left(\operatorname{rot}, \Im_{n}^{(r)}\right)
$$

are isomorphic.

Acknowledgements. The first author was partly supported by the Swedish Research Council (Vetenskapsrådet), grant 201505308. The second and third authors were supported by the Austrian Science Fund (FWF): P 29275. The second author is a recipient of a DOC Fellowship of the Austrian Academy of Sciences.

Conflict of Interest: None.

\section{References}

[AA19] P. Alexandersson and N. Amini, 'The cone of cyclic sieving phenomena', Discrete Math. 342(6) (2019), $1581-1601$. doi:10.1016/j.disc.2019.01.037.

[ALP19] P. Alexandersson, S. Linusson and S. Potka, 'The cyclic sieving phenomenon on circular Dyck paths', Electron. J. Combin. 26 (2019), 1-32. doi:10.37236/8720.

[AU20] P. Alexandersson and J. Uhlin, 'Cyclic sieving, skew Macdonald polynomials and Schur positivity', Algebr. Comb. 3(4) (2020), 913-939. doi:10.5802/alco.123.

[BMS14] M. Bennett, B. Madill and A. Stokke, 'Jeu-de-taquin promotion and a cyclic sieving phenomenon for semistandard hook tableaux’, Discrete Math. 319 (2014), 62-67. doi:10.1016/j.disc.2013.11.024.

[Bra41] R. Brauer, 'On the connection between the ordinary and the modular characters of groups of finite order', Ann. of Math. (2) 42 (1941), 926-935. doi:10.2307/1968774.

[BRS08] H. Barcelo, V. Reiner and D. Stanton, 'Bimahonian distributions', J. Lond. Math. Soc. (2) 77(3) (2008), $627-646$. doi:10.1112/jlms/jdn004.

[Dés83] J. Désarménien, 'Fonctions symétriques associées à des suites classiques de nombres', Ann. Sci. Éc. Norm. Supér. (4) 16(2) (1983), 271-304. doi:https://doi.org/10.24033/asens.1449.

[FK13] B. Fontaine and J. Kamnitzer, 'Cyclic sieving, rotation, and geometric representation theory', Selecta Math. (N.S.) 20(2) (2013), 609-625. doi:10.1007/s00029-013-0144-4.

[FL97] S. V. Fomin and N. Lulov, 'On the number of rim hook tableaux', J. Math. Sci. (N.Y.) 87(6) (1997), 4118-4123. doi:10.1007/bf02355806.

[JK84] G. James and A. Kerber, The Representation Theory of the Symmetric Group (Cambridge University Press, Cambridge, 1984). doi:10.1017/cbo9781107340732.

[Kov82] L. G. Kovács, 'The permutation lemma of Richard Brauer', Bull. Lond. Math. Soc. 14(2) (1982), 127-128. A letter to C. W. Curtis. doi:10.1112/blms/14.2.127. 
[Kup96] G. Kuperberg, 'Spiders for rank 2 Lie algebras', Comm. Math. Phys. 180(1) (1996), 109-151. doi:10.1007/bf02101184.

[LLT97] A. Lascoux, B. Leclerc and J.-Y. Thibon, 'Ribbon tableaux, Hall-Littlewood functions, quantum affine algebras and unipotent varieties', J. Math. Phys 38 (1997), 1041-1068.

[Mac95] I. G. Macdonald, Symmetric Functions and Hall Polynomials, second edn, Oxford Mathematical Monographs, (The Clarendon Press, New York, 1995). With contributions by A. Zelevinsky.

[OP20] Y.-T.Oh and E. Park, ' $q$-dimensions of highest weight crystals and cyclic sieving phenomenon', Preprint, 2020, arXiv:2008.03025.

[Pak00] I. Pak, 'Ribbon tile invariants', Trans. Amer. Math. Soc. 352(12) (2000), 5525-5562. doi:10.1090/s0002 -9947-00-02666-0.

[Pfa20] S. Pfannerer, In preparation.

[PRW20] S. Pfannerer, M. Rubey and B. W. Westbury, 'Promotion on oscillating and alternating tableaux and rotation of matchings and permutations', Algebr. Comb. 3(1) (2020), 107-141. doi:10.5802/alco.87.

[Pur13] K. Purbhoo, 'Wronskians, cyclic group actions, and ribbon tableaux', Trans. Amer. Math. Soc. 365(4) (2013), 1977-2030. URL: http://www.jstor.org/stable/23513431.

[Rhe12] D. Rhee, Cyclic Sieving Phenomenon of Promotion on Rectangular Tableaux (2012), M.S. thesis, University of Waterloo, Waterloo, Ontario, Canada. URL: https://pdfs.semanticscholar.org/ c7e0/60d4db5fa5a381da333678054288ed6e97a4.pdf.

[Rho10a] B. Rhoades, 'Cyclic sieving, promotion, and representation theory', J. Combin. Theory Ser. A 117(1) (2010), 38-76. doi:10.1016/j.jcta.2009.03.017.

[Rho10b] B. Rhoades, 'Hall-Littlewood polynomials and fixed point enumeration', Discrete Math. 310(4) (2010), 869-876. doi:10.1016/j.disc.2009.10.003.

[Rob55] H. Robbins, 'A remark on Stirling's formula', Amer. Math. Monthly 62(1) (1955), 26 . doi:10.2307/2308012.

[RSW04] V. Reiner, D. Stanton and D. E. White, 'The cyclic sieving phenomenon', J. Combin. Theory Ser. A 108(1) (2004), 17-50. doi:10.1016/j.jcta.2004.04.009.

[RW14] M. Rubey and B. W. Westbury, 'A combinatorial approach to classical representation theory', Preprint, 2014, arXiv: 1408.3592 .

[Spr74] T. A. Springer, 'Regular elements of finite reflection groups', Invent. Math. 25(2) (1974), 159-198. doi:10.1007/ bf01390173.

[SSW03] A. Schilling, M. Shimozono and D. E. White, 'Branching formula for $q$-Littlewood-Richardson coefficients', $A d v$. Appl. Math. 30(1-2) (2003), 258-272. doi:10.1016/s0196-8858(02)00535-3.

[SSW11] B. E. Sagan, J. Shareshian and M. L. Wachs, 'Eulerian quasisymmetric functions and cyclic sieving', Adv. Appl. Math. 46(1) (2011), 536-562. doi:10.1016/j.aam.2010.01.013.

[Sta01] R. P. Stanley, Enumerative Combinatorics: Volume 2, first edn, (Cambridge University Press, Cambridge, 2001). doi:10.1017/CBO9780511609589.

[Ste87] J. R. Stembridge, 'Rational tableaux and the tensor algebra of $\mathrm{gl}_{n}$ ', J. Combin. Theory Ser. A 46(1) (1987), 79-120. doi:10.1016/0097-3165(87)90077-x.

[Ste96] J. R. Stembridge, 'Canonical bases and self-evacuating tableaux', Duke Math. J. 82(3) (1996), 585-606. doi:10.1215/s0012-7094-96-08224-1.

[Wes16] B. W. Westbury, 'Invariant tensors and the cyclic sieving phenomenon', Electron. J. Combin. 23(4) (2016), 1-40. doi: $10.37236 / 4569$.

[Wes19] B. W. Westbury, 'Interpolating between promotion and the long cycle', Preprint, 2019, arXiv:1906.07146.

[Whi83] D. E. White, 'A bijection proving orthogonality of the characters of $S_{n}$ ', Adv. Math. 50(2) (1983), 160-186. doi:10.1016/0001-8708(83)90038-5. 